\title{
Two-loop scale-invariant scalar potential and quantum effective operators
}

\author{
D. M. Ghilencea ${ }^{1,2, a}$, Z. Lalak ${ }^{3, b}$, P. Olszewski ${ }^{3, c}$ \\ ${ }^{1}$ Theoretical Physics Department, National Institute of Physics and Nuclear Engineering (IFIN-HH), 077125 Bucharest, Romania \\ 2 Theory Division, CERN, 1211 Geneva 23, Switzerland \\ ${ }^{3}$ Faculty of Physics, Institute of Theoretical Physics, University of Warsaw, ul. Pasteura 5, 02-093 Warsaw, Poland
}

Received: 19 August 2016 / Accepted: 2 November 2016 / Published online: 29 November 2016

(C) The Author(s) 2016. This article is published with open access at Springerlink.com

\begin{abstract}
Spontaneous breaking of quantum scale invariance may provide a solution to the hierarchy and cosmological constant problems. In a scale-invariant regularization, we compute the two-loop potential of a Higgs-like scalar $\phi$ in theories in which scale symmetry is broken only spontaneously by the dilaton $(\sigma)$. Its $\operatorname{VEV}\langle\sigma\rangle$ generates the DR subtraction scale $(\mu \sim\langle\sigma\rangle)$, which avoids the explicit scale symmetry breaking by traditional regularizations (where $\mu=$ fixed scale). The two-loop potential contains effective operators of non-polynomial nature as well as new corrections, beyond those obtained with explicit breaking ( $\mu=$ fixed scale). These operators have the form $\phi^{6} / \sigma^{2}, \phi^{8} / \sigma^{4}$, etc., which generate an infinite series of higher dimensional polynomial operators upon expansion about $\langle\sigma\rangle \gg\langle\phi\rangle$, where such hierarchy is arranged by one initial, classical tuning. These operators emerge at the quantum level from evanescent interactions $(\alpha \epsilon)$ between $\sigma$ and $\phi$ that vanish in $d=4$ but are required by classical scale invariance in $d=4-2 \epsilon$. The Callan-Symanzik equation of the two-loop potential is respected and the two-loop beta functions of the couplings differ from those of the same theory regularized with $\mu=$ fixed scale. Therefore the running of the couplings enables one to distinguish between spontaneous and explicit scale symmetry breaking.
\end{abstract}

\section{Introduction}

Theories with scale symmetry [1,2] may provide a solution to the hierarchy and cosmological constant problems. But scale symmetry is not a symmetry of the real world, therefore it must be broken. In this work we discuss theories with

\footnotetext{
a e-mail: dumitru.ghilencea@ cern.ch

b e-mail: zygmunt.lalak@fuw.edu.pl

c e-mail: pawel.olszewski@fuw.edu.pl
}

scale invariance at the classical and quantum level that is broken only spontaneously. This is important since in a classical scale invariant theory, quantum calculations usually break this symmetry explicitly due to the presence of the subtraction (renormalization) scale $(\mu)$. This scale is introduced to regularize the loop integrals, regardless of the regularization method: dimensional regularization(DR), Pauli-Villars, etc., and its simple presence breaks explicitly this symmetry.

It is well known, however, how to avoid this problem by using a subtraction scale that is generated spontaneously, as the vacuum expectation value (VEV) of a scalar field $\sigma[3,4]$. This field is the Goldstone mode of scale symmetry (dilaton) and then $\mu=z\langle\sigma\rangle$, where $z$ is a dimensionless parameter. But before (spontaneous) scale symmetry breaking, with a field-dependent subtraction function $\mu(\sigma)=z \sigma$, there is no scale in the theory. One can use this idea to compute quantum corrections to the scalar potential of a theory with a Higgslike scalar $\phi$ and dilaton $\sigma$ and obtain a scale-invariant result at one loop [5-9] with a flat direction and spontaneous scale symmetry breaking. Although the result is scale invariant at the quantum level, the couplings still run with the momentum scale $[7,8,10] .^{1}$

To illustrate some of these ideas, consider a scale-invariant theory in $d=4$

$L=\frac{1}{2} \partial_{\mu} \phi \partial^{\mu} \phi+\frac{1}{2} \partial_{\mu} \sigma \partial^{\mu} \sigma-V(\phi, \sigma)$

where $\phi$ is a Higgs-like scalar and $\sigma$ is a dilaton. In such a theory $V$ has a form

$V(\phi, \sigma)=\sigma^{4} W(\phi / \sigma)$.

In this paper we assume that we have spontaneous breaking of this symmetry, hence $\langle\sigma\rangle \neq 0$. We do not detail how $\sigma$

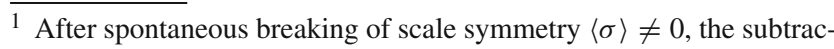
tion scale $\mu(\langle\sigma\rangle)$ and all other masses/VEVs of the theory are generated, proportional to $\langle\sigma\rangle$.
} 
acquires a VEV (expected to be large $\langle\sigma\rangle \sim M_{\text {Planck }}$ ) but search for solutions with $\langle\sigma\rangle \neq 0$. Then the two minimum conditions $\partial V / \partial \phi=\partial V / \partial \sigma=0$ become

$W^{\prime}\left(x_{0}\right)=W\left(x_{0}\right)=0, \quad x_{0} \equiv \frac{\langle\phi\rangle}{\langle\sigma\rangle} ; \quad\langle\sigma\rangle,\langle\phi\rangle \neq 0$.

At a given order $n$ in perturbation theory, one condition, say $W^{\prime}\left(x_{0}\right)=0$, fixes the ratio $x_{0} \equiv\langle\phi\rangle /\langle\sigma\rangle$ in terms of the (dimensionless) couplings of the theory. The second condition, $W\left(x_{0}\right)=0$, leads to vanishing vacuum energy $V(\langle\phi\rangle,\langle\sigma\rangle)=0$ and fixes a relation among the couplings, corrected to that order $(n)$ in perturbation theory from its version in the lower perturbation order $(n-1)$. If these two equations have a solution $x_{0}$, then the system has a flat direction (Goldstone) in the plane $(\phi, \sigma)$ with $\phi / \sigma=x_{0}$. Then a massless state exists (dilaton) at this order. This is true provided that quantum corrections do not break explicitly the scale symmetry (otherwise, Eq. (2) is not valid due to the presence of the "usual" DR scale $\mu$ ). With a scale-invariant regularization, it is possible to keep these properties ( $V=0$, a flat direction, etc.) and study spontaneously broken quantum scale invariance.

Why is this interesting? One reason is that this answers the question of Bardeen [11] on the mass hierarchy. The Standard Model (SM) with a vanishing classical Higgs mass term is scale invariant and there is no mass hierarchy (ignoring gravity, as here ${ }^{2}$ ). If quantum calculations preserve this symmetry, via a scale-invariant regularization, one can avoid a hierarchy problem and the fine-tuning of the Higgs selfcoupling and keep it light relative to the high scale (physical mass of a new state) generated by $\langle\sigma\rangle \neq 0$. One can arrange that $x_{0}=\langle\phi\rangle /\langle\sigma\rangle \ll 1$ by a single classical tuning of the (ratio of the) couplings of the theory [20]. The hierarchy $m_{\text {Higgs }}^{2} \sim\langle\phi\rangle^{2} \ll\langle\sigma\rangle^{2}$ is maintained at one loop [5-9,20,21] and probably beyond it, due to the spontaneousonly scale symmetry breaking. The only difference from the usual SM is the presence of a massless dilaton in addition to the SM spectrum. Also, the solution $x_{0}$ is related to the (minimum) condition $V=0$. This suggests that in spontaneously broken quantum scale-invariant theories any fine tuning is related to vacuum energy tuning at the same order of perturbation.

With this motivation, in this paper we extend the above results. We consider a classically scale-invariant theory of $\phi$ and $\sigma$ and compute at two loops the scalar potential and the running of the couplings, in a scale-invariant regularization. We find that starting from two loops, the running of the couplings differs from that in the same theory of $\phi, \sigma$ regularized with $\mu=$ constant. We show that effective non-polynomial operators like $\phi^{6} / \sigma^{2}, \phi^{8} / \sigma^{4}$, are generated as two-loop counterterms. If expanded about the ground state, these operators

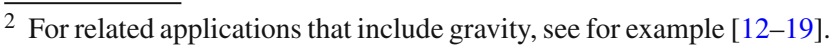

generate an infinite series of polynomial terms, showing the non-renormalizability of the theory. The Callan-Symanzik equation of the potential is verified at two loops. The results are useful for phenomenology, e.g. to study a scale-invariant version of the SM (+dilaton).

\section{One-loop potential}

We first review the one-loop potential $[8,9]$. Consider the classical potential ${ }^{3}$

$V=\frac{\lambda_{\phi}}{4 !} \phi^{4}+\frac{\lambda_{m}}{4} \phi^{2} \sigma^{2}+\frac{\lambda_{\sigma}}{4 !} \sigma^{4}$.

Spontaneous scale symmetry breaking $\langle\sigma\rangle \neq 0$ requires two conditions (Eq. (3)) be met:

$$
\begin{aligned}
& 9 \lambda_{m}^{2}=\lambda_{\phi} \lambda_{\sigma}+\text { loops, }\left(\lambda_{m}<0\right), \\
& \text { and } \quad x_{0}^{2} \equiv \frac{\langle\phi\rangle^{2}}{\langle\sigma\rangle^{2}}=-\frac{3 \lambda_{m}}{\lambda_{\phi}}+\text { loops }
\end{aligned}
$$

A massless (Goldstone) state exists corresponding to a flat direction $\phi=x_{0} \sigma$ with $V_{\min }=0$. With $\phi$ being Higgslike, scale symmetry breaking implies electroweak symmetry breaking.

To compute quantum corrections in $d=4-2 \epsilon$, the scalar potential is modified to $\tilde{V}=\mu^{2 \epsilon} V$ to ensure dimensionless quartic couplings, with $\mu$ the "usual" DR subtraction scale. General principles ${ }^{4}$ suggest that the subtraction function $\mu(\sigma)$ depend on the dilaton only [8] and generate the subtraction scale $\mu(\langle\sigma\rangle)$ after spontaneous scale symmetry breaking; $\mu(\sigma)$ is then identified on dimensional grounds (using $[\mu]=1,[\sigma]=(d-2) / 2$ ). Then the scale-invariant potential in $d=4-2 \epsilon$ and $\mu(\sigma)$ become

$$
\tilde{V}(\phi, \sigma)=\mu(\sigma)^{2 \epsilon} V(\phi, \sigma), \quad \mu(\sigma)=z \sigma^{1 /(1-\epsilon)},
$$

where $z$ is an arbitrary dimensionless parameter. ${ }^{5}$ The oneloop result is

$V_{1}=\tilde{V}-\frac{i}{2} \int \frac{\mathrm{d}^{d} p}{(2 \pi)^{d}} \operatorname{Tr} \ln \left[p^{2}-\tilde{V}_{i j}+i \varepsilon\right]$.

Here $\tilde{V}_{i j}=\partial^{2} \tilde{V} / \partial s_{i} \partial s_{j} \quad(i, j=1,2), s_{1}=\phi, s_{2}=\sigma$, and similar for $V_{i j}=\partial^{2} V / \partial s_{i} \partial s_{j}$. Also $\tilde{V}_{i j}=\mu^{2 \epsilon}\left[V_{i j}+\right.$ $\left.2 \epsilon \mu^{-2} N_{i j}\right]+\mathcal{O}\left(\epsilon^{2}\right)$, where

\footnotetext{
3 In principle one can also include higher dimensional terms like $\phi^{6} / \sigma^{2}, \phi^{8} / \sigma^{4}$, etc. $(\langle\sigma\rangle \neq 0)$; see later.

4 They require quantum interactions between $\phi$ and $\sigma$ vanish in their classically decoupling limit $\lambda_{m}=0$.

5 The parameter $z$ plays a special role in the Callan-Symanzik equation, see later.
} 


$$
\begin{aligned}
N_{i j} \equiv & \mu\left\{\frac{\partial \mu}{\partial s_{i}} \frac{\partial V}{\partial s_{j}}+\frac{\partial \mu}{\partial s_{j}} \frac{\partial V}{\partial s_{i}}\right\} \\
& +\left\{\mu \frac{\partial^{2} \mu}{\partial s_{i} \partial s_{j}}-\frac{\partial \mu}{\partial s_{i}} \frac{\partial \mu}{\partial s_{j}}\right\} V, \quad i, j=1,2 .
\end{aligned}
$$

Then

$$
\begin{aligned}
V_{1}= & \mu(\sigma)^{2 \epsilon}\left\{V-\frac{1}{64 \pi^{2}}\left[\sum_{s=\phi, \sigma} M_{s}^{4}\left(\frac{1}{\epsilon}-\ln \frac{M_{s}^{2}(\phi, \sigma)}{c_{0} \mu^{2}(\sigma)}\right)\right.\right. \\
& \left.\left.+\frac{4\left(V_{i j} N_{j i}\right)}{\mu^{2}(\sigma)}\right]\right\}
\end{aligned}
$$

with an implicit sum over $i, j$ and with $c_{0}=4 \pi e^{3 / 2-\gamma_{E}}$. The one-loop Lagrangian is

$L_{1}=\frac{1}{2}\left(\partial_{\mu} \phi\right)^{2}+\frac{1}{2}\left(\partial_{\mu} \sigma\right)^{2}-V_{1}$.

Above, $M_{s}^{2}$ denotes the field-dependent eigenvalues of the matrix $V_{i j}$. The poles in $L_{1}$ are canceled by adding the counterterm Lagrangian $\delta L_{1}$ found using the expression of $M_{s}^{2}$ :

$$
\begin{aligned}
\delta L_{1} \equiv & -\delta V_{1}=-\mu(\sigma)^{2 \epsilon}\left\{\frac{1}{4 !}\left(Z_{\lambda_{\phi}}-1\right) \lambda_{\phi} \phi^{4}\right. \\
& \left.+\frac{1}{4}\left(Z_{\lambda_{m}}-1\right) \lambda_{m} \phi^{2} \sigma^{2}+\frac{1}{4 !}\left(Z_{\lambda_{\sigma}}-1\right) \lambda_{\sigma} \sigma^{4}\right\}
\end{aligned}
$$

with

$$
\begin{aligned}
& Z_{\lambda_{\phi}}=1+\frac{3}{2 \kappa \epsilon}\left(\lambda_{\phi}+\lambda_{m}^{2} / \lambda_{\phi}\right), \\
& Z_{\lambda_{m}}=1+\frac{1}{2 \kappa \epsilon}\left(\lambda_{\phi}+\lambda_{\sigma}+4 \lambda_{m}\right), \\
& Z_{\lambda_{\sigma}}=1+\frac{3}{2 \kappa \epsilon}\left(\lambda_{\sigma}+\lambda_{m}^{2} / \lambda_{\sigma}\right), \quad \kappa=(4 \pi)^{2} .
\end{aligned}
$$

$Z_{\lambda}$ 's are identical to their counterparts computed in the same theory regularized with $\mu=$ constant (when scale symmetry is broken explicitly). The one-loop potential becomes

$$
\begin{aligned}
U_{1}=V & +V^{(1)}+V^{(1, n)}, \\
V^{(1)} \equiv & \frac{1}{64 \pi^{2}} \sum_{s=\phi, \sigma} M_{s}^{4}(\phi, \sigma)\left[\ln \frac{M_{s}^{2}(\phi, \sigma)}{\mu^{2}(\sigma)}-\frac{3}{2}\right], \\
V^{(1, n)} \equiv & \frac{1}{48 \kappa}\left[\lambda_{\phi} \lambda_{m} \frac{\phi^{6}}{\sigma^{2}}-\left(16 \lambda_{\phi} \lambda_{m}+18 \lambda_{m}^{2}-\lambda_{\phi} \lambda_{\sigma}\right) \phi^{4}\right. \\
& \left.\quad-\left(48 \lambda_{m}+25 \lambda_{\sigma}\right) \lambda_{m} \phi^{2} \sigma^{2}-7 \lambda_{\sigma}^{2} \sigma^{4}\right]
\end{aligned}
$$

The potential simplifies further if we use the tree-level relation (5) among $\lambda_{s}(s=\phi, m, \sigma)$ that ensures the spontaneous scale symmetry breaking. $U_{1}$ is scale symmetric and a flat direction exists also at the quantum level. $V_{1}^{(1, n)}$ is a new, finite one-loop correction, independent of the parameter $z$; it contains a non-polynomial term $\phi^{6} / \sigma^{2}$ that can be Taylorexpanded about $\langle\phi\rangle,\langle\sigma\rangle \neq 0 . V^{(1, \mathrm{n})} \rightarrow 0$ in the classical decoupling limit $\lambda_{m} \rightarrow 0$. The Coleman-Weinberg term is also present, with $\mu \rightarrow \mu(\sigma)$ and thus depends on $z$. This dependence replaces the "traditional" dependence of $V^{(1)}$ on the subtraction scale in theories regularized with $\mu=$ constant. But physics should be independent of this parameter, which means that $U_{1}$ must respect the Callan-Symanzik equation: $\mathrm{d} U_{1} / \mathrm{d} \ln z=0$ [10].

To check the Callan-Symanzik equation, we need the beta functions of the couplings which run with the momentum, even in scale-invariant theories $[7,10]$. These are computed from the condition $\mathrm{d}\left(\mu(\sigma)^{2 \epsilon} \lambda_{j} Z_{\lambda_{j}}\right) / \mathrm{d} \ln z=0$ ( $j$ : fixed), since the bare coupling is independent of $z$. The result is identical to that in a theory regularized with $\mu=$ constant:

$\beta_{\lambda_{\phi}}^{(1)} \equiv \frac{\mathrm{d} \lambda_{\phi}}{\mathrm{d} \ln z}=\frac{3}{\kappa}\left(\lambda_{\phi}^{2}+\lambda_{m}^{2}\right)$,

$\beta_{\lambda_{m}}^{(1)} \equiv \frac{\mathrm{d} \lambda_{m}}{\mathrm{~d} \ln z}=\frac{1}{\kappa}\left(\lambda_{\phi}+4 \lambda_{m}+\lambda_{\sigma}\right) \lambda_{m}$,

$\beta_{\lambda_{\sigma}}^{(1)} \equiv \frac{\mathrm{d} \lambda_{\sigma}}{\mathrm{d} \ln z}=\frac{3}{\kappa}\left(\lambda_{m}^{2}+\lambda_{\sigma}^{2}\right)$.

The Callan-Symanzik equation at one loop is

$$
\begin{aligned}
& \frac{\mathrm{d} U_{1}\left(\lambda_{j}, z\right)}{\mathrm{d} \ln z}=\left(\beta_{\lambda_{j}}^{(1)} \frac{\partial}{\partial \lambda_{j}}+z \frac{\partial}{\partial z}\right) U_{1}\left(\lambda_{j}, z\right)=\mathcal{O}\left(\lambda_{j}^{3}\right), \\
& \quad(\text { sum over } j=\phi, m, \sigma) .
\end{aligned}
$$

Equation (19) is easily verified with the above results for the beta functions. The one-loop $U_{1}$ can be used for phenomenology of a scale-invariant version of the SM extended by the dilaton [8].

\section{Two-loop potential}

\subsection{New poles in the two-loop potential}

To compute the two-loop potential we use the background field expansion method about $\phi, \sigma$. We Taylor-expand $\tilde{V}$ about these values

$$
\begin{aligned}
& \tilde{V}(\phi+\delta \phi, \sigma+\delta \sigma)=V(\phi, \sigma)+\tilde{V}_{j} s_{j}+\frac{1}{2} \tilde{V}_{j k} s_{j} s_{k} \\
& +\frac{1}{3 !} \tilde{V}_{i j k} s_{i} s_{j} s_{k}+\frac{1}{4 !} \tilde{V}_{i j k l} s_{i} s_{j} s_{k} s_{l}+\cdots
\end{aligned}
$$

where the subscripts $i, j, k, l$ of $\tilde{V}_{i j \ldots}$ denote derivatives of $\tilde{V}$ w.r.t. fields of the set $\{\phi, \sigma\}_{j}$; with $i, j, k, l=1,2$. Also $s_{1}=$ $\delta \phi, s_{2}=\delta \sigma$ are field fluctuations. Notice that there are new, evanescent interactions $(\alpha \epsilon)$ in vertices $\tilde{V}_{i j k \ldots}$ generated by Eq. (6) that impact on the loop corrections. The two-loop diagrams are presented below. Let us first denote

$V_{2}=V_{2}^{a}+V_{2}^{b}+V_{2}^{c}$ 
Then

$$
\begin{aligned}
& V_{2}^{a}= \frac{i}{12} \bigcirc=\frac{i}{12} \tilde{V}_{i j k} \tilde{V}_{l m n} \int \frac{d^{d} p}{(2 \pi)^{d}} \int \frac{d^{d} q}{(2 \pi)^{d}}\left(\tilde{D}_{p}^{-1}\right)_{i l}\left(\tilde{D}_{q}^{-1}\right)_{j m}\left(\tilde{D}_{p+q}^{-1}\right)_{k n} \\
&=\frac{\mu(\sigma)^{2 \epsilon}}{\epsilon^{2}} \frac{1}{16 \kappa^{2}}\left[\phi^{4}\left(\lambda_{\phi}^{3}+\lambda_{\phi} \lambda_{m}^{2}+2 \lambda_{m}^{3}\right)+\sigma^{4}\left(2 \lambda_{m}^{3}+\lambda_{m}^{2} \lambda_{\sigma}+\lambda_{\sigma}^{3}\right)\right. \\
&\left.+\phi^{2} \sigma^{2}\left(\lambda_{\phi}^{2} \lambda_{m}+6 \lambda_{\phi} \lambda_{m}^{2}+10 \lambda_{m}^{3}+6 \lambda_{m}^{2} \lambda_{\sigma}+\lambda_{m} \lambda_{\sigma}^{2}\right)\right]+\mathcal{O}(1 / \epsilon) .
\end{aligned}
$$

Also

$$
\begin{gathered}
V_{2}^{b=} \frac{i}{8} \bigcirc=\frac{i}{8} \tilde{V}_{i j k l}\left[\int \frac{d^{d} p}{(2 \pi)^{d}}\left(\tilde{D}_{p}^{-1}\right)_{i j}\right]\left[\int \frac{d^{d} q}{(2 \pi)^{d}}\left(\tilde{D}_{q}^{-1}\right)_{k l}\right] \\
=\frac{\mu(\sigma)^{2 \epsilon}}{\epsilon^{2}} \frac{1}{32 \kappa^{2}}\left[\phi^{4}\left(\lambda_{\phi}^{3}+2 \lambda_{\phi} \lambda_{m}^{2}+\lambda_{m}^{2} \lambda_{\sigma}\right)+\sigma^{4}\left(\lambda_{\phi} \lambda_{m}^{2}+2 \lambda_{m}^{2} \lambda_{\sigma}+\lambda_{\sigma}^{3}\right)\right. \\
\left.+2 \lambda_{m} \phi^{2} \sigma^{2}\left(\lambda_{\phi}^{2}+9 \lambda_{m}^{2}+\lambda_{\phi} \lambda_{\sigma}+\lambda_{\sigma}^{2}\right)\right]+\mathcal{O}(1 / \epsilon)
\end{gathered}
$$

and finally

$$
\begin{aligned}
V_{2}^{c} & =\frac{i}{2} \bigcirc=\frac{i}{2}\left(\delta V_{1}\right)_{i j} \int \frac{d^{d} p}{(2 \pi)^{d}}\left(\tilde{D}_{p}^{-1}\right)_{i j} \\
& =\frac{\mu(\sigma)^{2 \epsilon}}{\epsilon^{2}} \frac{(-1)}{16 \kappa^{2}}\left[\phi^{4}\left(3 \lambda_{\phi}^{3}+4 \lambda_{\phi} \lambda_{m}^{2}+4 \lambda_{m}^{3}+\lambda_{m}^{2} \lambda_{\sigma}\right)+\sigma^{4}\left(\lambda_{\phi} \lambda_{m}^{2}+4 \lambda_{m}^{3}+4 \lambda_{m}^{2} \lambda_{\sigma}+3 \lambda_{\sigma}^{3}\right)\right. \\
& \left.+\phi^{2} \sigma^{2}\left(4 \lambda_{\phi}^{2} \lambda_{m}+12 \lambda_{\phi} \lambda_{m}^{2}+38 \lambda_{m}^{3}+2 \lambda_{\phi} \lambda_{m} \lambda_{\sigma}+12 \lambda_{m}^{2} \lambda_{\sigma}+4 \lambda_{m} \lambda_{\sigma}^{2}\right)\right]+\mathcal{O}(1 / \epsilon) .
\end{aligned}
$$

These diagrams are computed using [22]; see also [23]. The propagators are given by the inverse of the matrix $\left(\tilde{D}_{p}\right)_{i j}=p^{2} \delta_{i j}-\tilde{V}_{i j}$. To simplify the calculation they can be re-written as $\left(\tilde{D}^{-1}\right)_{i j}=\tilde{a}_{i j} /\left(p^{2}-\tilde{V}_{p}\right)+\tilde{b}_{i j} /\left(p^{2}-\tilde{V}_{m}\right)$, with appropriate coefficients $\tilde{a}_{i j}$ and $\tilde{b}_{i j}$ and where $\tilde{V}_{p}, \tilde{V}_{m}$ $\left(\tilde{V}_{p}>\tilde{V}_{m}\right)$ denote the field-dependent masses, eigenvalues of the matrix $\tilde{V}_{i j}, i, j=\phi, \sigma$. Note that $\tilde{V}_{i j}, \tilde{V}_{p}, \tilde{V}_{m}, \tilde{a}_{i j}$, $\tilde{b}_{i j}$, and also $\tilde{V}_{i j k l}, \tilde{V}_{i j k}$ contain positive powers of $\epsilon$; this is relevant for the above calculation, since they contribute to the finite and $1 / \epsilon$ parts of the potential. Their form is detailed in Appendices B and C.

One notices that the poles $1 / \epsilon^{2}$ in $V_{2}^{a, b, c}$ are identical to those in the theory regularized with $\mu=$ constant. This is expected for this leading singularity, but this is not true for their sub-leading one $(1 / \epsilon)$ or for their finite part (see later). The long expressions $\mathcal{O}(1 / \epsilon)$ and $\mathcal{O}\left(\epsilon^{0}\right)$ of each diagram $V_{2}^{a, b, c}$ are not shown here. The sum of these diagrams gives

$$
\begin{aligned}
V_{2}= & \frac{\mu(\sigma)^{2 \epsilon}}{\epsilon^{2}} \frac{(-1)}{32 \kappa^{2}}\left[\phi ^ { 4 } \left(3 \lambda_{\phi}^{3}+4 \lambda_{\phi} \lambda_{m}^{2}\right.\right. \\
& \left.+4 \lambda_{m}^{3}+\lambda_{m}^{2} \lambda_{\sigma}\right)+\sigma^{4}\left(3 \lambda_{\sigma}^{3}+\lambda_{\phi} \lambda_{m}^{2}+4 \lambda_{m}^{3}+4 \lambda_{m}^{2} \lambda_{\sigma}\right) \\
& +\phi^{2} \sigma^{2}\left(4 \lambda_{\phi}^{2} \lambda_{m}+12 \lambda_{\phi} \lambda_{m}^{2}+38 \lambda_{m}^{3}+2 \lambda_{\phi} \lambda_{m} \lambda_{\sigma}\right. \\
& \left.\left.+12 \lambda_{m}^{2} \lambda_{\sigma}+4 \lambda_{m} \lambda_{\sigma}^{2}\right)\right] \\
& +\frac{\mu(\sigma)^{2 \epsilon}}{\epsilon} \frac{1}{16 \kappa^{2}}\left[\phi^{4}\left(\lambda_{\phi}^{3}+\lambda_{\phi} \lambda_{m}^{2}+2 \lambda_{m}^{3}\right)\right. \\
& +\sigma^{4}\left(2 \lambda_{m}^{3}+\lambda_{m}^{2} \lambda_{\sigma}+\lambda_{\sigma}^{3}\right) \\
& \left.+\phi^{2} \sigma^{2}\left(\lambda_{\phi}^{2} \lambda_{m}+6 \lambda_{\phi} \lambda_{m}^{2}+10 \lambda_{m}^{3}+6 \lambda_{m}^{2} \lambda_{\sigma}+\lambda_{m} \lambda_{\sigma}^{2}\right)\right] \\
& +V_{2}^{1 / \epsilon}+V^{(2)}+V^{(2, n)} .
\end{aligned}
$$

Here $V^{(2)}$ and $V^{(2, n)}$ are $\mathcal{O}\left(\epsilon^{0}\right)$ i.e. finite quantum corrections presented in Appendix B. $V_{2}^{1 / \epsilon}=\mathcal{O}(1 / \epsilon)$ is a new term that contains $1 / \epsilon$ poles not present in the theory regularized with $\mu=$ constant; its origin is due to evanescent interactions $(\propto \epsilon)$, which "meet" $1 / \epsilon^{2}$ poles, thus giving $1 / \epsilon$ terms. One finds 


$$
\begin{aligned}
V_{2}^{1 / \epsilon}= & \frac{\mu(\sigma)^{2 \epsilon}}{16 \kappa^{2} \epsilon}\left[\phi ^ { 4 } \left(\frac{20}{3} \lambda_{\phi}^{2} \lambda_{m}+\frac{7}{6} \lambda_{\phi} \lambda_{m}^{2}-2 \lambda_{m}^{3}-\frac{1}{2} \lambda_{\phi}^{2} \lambda_{\sigma}\right.\right. \\
& \left.-\frac{4}{3} \lambda_{\phi} \lambda_{m} \lambda_{\sigma}+\frac{7}{12} \lambda_{m}^{2} \lambda_{\sigma}+\frac{1}{4} \lambda_{\phi} \lambda_{\sigma}^{2}\right) \\
& +\phi^{2} \sigma^{2}\left(8 \lambda_{\phi} \lambda_{m}^{2}+\frac{41}{2} \lambda_{m}^{3}+\lambda_{\phi} \lambda_{m} \lambda_{\sigma}\right. \\
& \left.+\frac{43}{3} \lambda_{m}^{2} \lambda_{\sigma}+\frac{1}{2} \lambda_{m} \lambda_{\sigma}^{2}\right) \\
& +\sigma^{4}\left(4 \lambda_{m}^{3}+\frac{1}{3} \lambda_{m}^{2} \lambda_{\sigma}+\frac{7}{4} \lambda_{\sigma}^{3}\right) \\
& +\frac{\phi^{6}}{\sigma^{2}}\left(-\frac{7}{6} \lambda_{\phi}^{2} \lambda_{m}+\frac{7}{3} \lambda_{\phi} \lambda_{m}^{2}-\frac{1}{6} \lambda_{\phi} \lambda_{m} \lambda_{\sigma}\right) \\
& \left.-\frac{1}{4} \lambda_{\phi} \lambda_{m}^{2} \frac{\phi^{8}}{\sigma^{4}}\right] .
\end{aligned}
$$

In addition to usual counterterms ( $\phi^{4}$, etc.), notice from Eq. (26) the need for non-polynomial counterterms $\phi^{6} / \sigma^{2}$ and $\phi^{8} / \sigma^{4}$ (see also $\phi^{6} / \sigma^{2}$ in Eq. (13)). The above two-loop results contribute to the Lagrangian (below $\rho^{\phi}, \rho^{\sigma}$ are wavefunction coefficients defined later)

$$
\begin{aligned}
L_{2}= & \frac{1}{2}\left(\frac{\rho^{\phi}}{\epsilon}+\text { finite }\right)\left(\partial_{\mu} \phi\right)^{2} \\
& +\frac{1}{2}\left(\frac{\rho^{\sigma}}{\epsilon}+\text { finite }\right)\left(\partial_{\mu} \sigma\right)^{2}-V_{2} .
\end{aligned}
$$

A counterterm $\delta L_{2}$ cancels the poles in the sum $L_{1}+L_{2}$ of Eqs. (10) and (27) up to two loops

$$
\begin{aligned}
\delta L_{2}= & \frac{1}{2}\left(Z_{\phi}-1\right)\left(\partial_{\mu} \phi\right)^{2}+\frac{1}{2}\left(Z_{\sigma}-1\right)\left(\partial_{\mu} \sigma\right)^{2} \\
& -\mu(\sigma)^{2 \epsilon}\left\{\left(Z_{\lambda_{\phi}}-1\right) \frac{\lambda_{\phi}}{4 !} \phi^{4}\right. \\
& +\left(Z_{\lambda_{m}}-1\right) \frac{\lambda_{m}}{4} \phi^{2} \sigma^{2}+\left(Z_{\lambda_{\sigma}}-1\right) \frac{\lambda_{\sigma}}{4 !} \sigma^{4} \\
& \left.+\left(Z_{\lambda_{6}}-1\right) \frac{\lambda_{6}}{6} \frac{\phi^{6}}{\sigma^{2}}+\left(Z_{\lambda_{8}}-1\right) \frac{\lambda_{8}}{8} \frac{\phi^{8}}{\sigma^{4}}\right\},
\end{aligned}
$$

where

$$
\begin{aligned}
& Z_{\lambda_{\phi}}=1+\frac{\delta_{0}^{\phi}}{\kappa \epsilon}+\frac{1}{\kappa^{2}}\left(\frac{\delta_{1}^{\phi}+v_{1}^{\phi}}{\epsilon}+\frac{\delta_{2}^{\phi}}{\epsilon^{2}}\right), \\
& Z_{\lambda_{m}}=1+\frac{\delta_{0}^{m}}{\kappa \epsilon}+\frac{1}{\kappa^{2}}\left(\frac{\delta_{1}^{m}+v_{1}^{m}}{\epsilon}+\frac{\delta_{2}^{m}}{\epsilon^{2}}\right), \\
& Z_{\lambda_{\sigma}}=1+\frac{\delta_{0}^{\sigma}}{\kappa \epsilon}+\frac{1}{\kappa^{2}}\left(\frac{\delta_{1}^{\sigma}+v_{1}^{\sigma}}{\epsilon}+\frac{\delta_{2}^{\sigma}}{\epsilon^{2}}\right), \\
& Z_{\lambda_{6}}=1+\frac{1}{\kappa^{2}} \frac{\nu_{1}^{6}}{\epsilon} ; \quad Z_{\lambda_{8}}=1+\frac{1}{\kappa^{2}} \frac{\nu_{1}^{8}}{\epsilon},
\end{aligned}
$$

where the one-loop $\delta_{0}^{\phi}, \delta_{0}^{m}, \delta_{0}^{\sigma}$ can be read from Eq. (12), while the two-loop coefficients $\delta_{k}^{s}, k=1,2, s=\phi, m, \sigma$, are shown in Appendix A. They are obtained by comparing $\delta L_{2}$ against $L_{2}$, using $V_{2}$ of Eq. (25). The coefficients $\delta_{k}^{s}$ are those of the theory regularized with $\mu=$ constant. However, there is an extra contribution from the coefficients $v_{1}^{s}, s=$ $\phi, m, \sigma, 6,8$ (see Appendix A), which is generated by the new poles $1 / \epsilon$ of $V_{2}^{1 / \epsilon}$. This new contribution brings about a correction to the two-loop beta functions of our theory, see later.

One can also show that the two-loop-corrected wavefunction coefficients have expressions similar to those in the theory regularized with $\mu=$ constant:

$$
\begin{array}{ll}
Z_{\phi}=1+\frac{\rho^{\phi}}{\kappa^{2} \epsilon}, & \rho^{\phi}=-\frac{1}{24}\left(\lambda_{\phi}^{2}+3 \lambda_{m}^{2}\right), \\
Z_{\sigma}=1+\frac{\rho^{\sigma}}{\kappa^{2} \epsilon}, & \rho^{\sigma}=-\frac{1}{24}\left(\lambda_{\sigma}^{2}+3 \lambda_{m}^{2}\right) .
\end{array}
$$

One often uses the notation $\gamma_{\phi}=-2 \rho^{\phi} / \kappa^{2}$ and $\gamma_{\sigma}=$ $-2 \rho^{\sigma} / \kappa^{2}$ for the anomalous dimensions.

\subsection{Two-loop beta functions}

With the above information, one obtains the two-loop beta functions. To this purpose, one uses that the "bare" couplings $\lambda_{j}^{B}$ below are independent of the parameter $z$ :

$\lambda_{\phi}^{B}=\mu(\sigma)^{2 \epsilon} \lambda_{\phi} Z_{\lambda_{\phi}} Z_{\phi}^{-2}$

$\lambda_{m}^{B}=\mu(\sigma)^{2 \epsilon} \lambda_{m} Z_{\lambda_{m}} Z_{\phi}^{-1} Z_{\sigma}^{-1}$,

$\lambda_{\sigma}^{B}=\mu(\sigma)^{2 \epsilon} \lambda_{\sigma} Z_{\lambda_{\sigma}} Z_{\sigma}^{-2}$,

$\lambda_{6}^{B}=\mu(\sigma)^{2 \epsilon} \lambda_{6} Z_{\lambda_{6}} Z_{\phi} Z_{\sigma}^{-3}$.

We thus require $(\mathrm{d} / \mathrm{d} \ln z) \lambda_{k}^{B}=0, k=\phi, m, \sigma, 6,8 .^{6}$ Taking the logarithm of the first expression in (31) and then the derivative with respect to $\ln z$, one obtains

$2 \epsilon+\frac{\beta_{\lambda_{\phi}}}{\lambda_{\phi}}+\sum_{j=\phi, m, \sigma} \beta_{\lambda_{j}} \frac{\mathrm{d}}{\mathrm{d} \lambda_{j}} \ln \left[Z_{\lambda_{\phi}} Z_{\phi}^{-2}\right]=0$

and similar expressions for the other couplings. Using the form of $Z^{\prime}$ s, one finds

$$
\begin{aligned}
\beta_{\lambda_{\phi}}= & -2 \epsilon \lambda_{\phi}+2 \lambda_{\phi} \sum_{j=\phi, m, \sigma} \lambda_{j} \frac{\mathrm{d}}{\mathrm{d} \lambda_{j}} \\
& \times\left(\frac{\delta_{0}^{\phi}}{\kappa}+\frac{\delta_{1}^{\phi}+v_{1}^{\phi}}{\kappa^{2}}-\frac{2 \rho^{\phi}}{\kappa^{2}}\right) .
\end{aligned}
$$

One easily obtains similar relations for $\beta_{\lambda_{m}}$ and $\beta_{\lambda_{\sigma}}$ (for $\beta_{\lambda_{\sigma}}$ just replace the sub-/super-script $\phi \rightarrow \sigma$ ). The difference in these beta functions from those in the same theory but regularized with $\mu=$ constant is the presence of a new contribution: $v_{1}^{\phi}\left(v_{1}^{m}, v_{1}^{\sigma}\right.$, respectively), which we identified in

6 We also include the effect of wavefunction renormalization of the subtraction function which requires replacing $\mu(\sigma)=z \sigma^{1 /(1-\epsilon)} \rightarrow$ $z\left(Z_{\sigma}^{1 / 2} \sigma\right)^{1 /(1-\epsilon)}$; however, this brings no correction in this order. 
Eqs. (29). Equation (33) is solved with particular attention to the $\epsilon$-dependent terms, to find at two loops

$$
\begin{aligned}
\beta_{\lambda_{\phi}}= & \frac{3}{\kappa}\left(\lambda_{\phi}^{2}+\lambda_{m}^{2}\right)-\frac{1}{\kappa^{2}}\left(\frac{17}{3} \lambda_{\phi}^{3}+5 \lambda_{\phi} \lambda_{m}^{2}+12 \lambda_{m}^{3}\right) \\
& +\beta_{\lambda_{\phi}}^{(2, \mathrm{n})}, \\
\beta_{\lambda_{m}}= & \frac{1}{\kappa}\left(\lambda_{\phi}+4 \lambda_{m}+\lambda_{\sigma}\right) \lambda_{m}-\frac{\lambda_{m}}{6 \kappa^{2}}\left(5 \lambda_{\phi}^{2}+36 \lambda_{\phi} \lambda_{m}\right. \\
& \left.+54 \lambda_{m}^{2}+36 \lambda_{m} \lambda_{\sigma}+5 \lambda_{\sigma}^{2}\right)+\beta_{\lambda_{m}}^{(2, \mathrm{n})}, \\
\beta_{\lambda_{\sigma}}= & \frac{3}{\kappa}\left(\lambda_{m}^{2}+\lambda_{\sigma}^{2}\right)-\frac{1}{\kappa^{2}}\left(12 \lambda_{m}^{3}+5 \lambda_{m}^{2} \lambda_{\sigma}+\frac{17}{3} \lambda_{\sigma}^{3}\right) \\
& +\beta_{\lambda_{\sigma}}^{(2, \mathrm{n})} .
\end{aligned}
$$

The "new" terms $\beta_{\lambda}^{(2, n)}$ on the r.h.s. are

$$
\begin{aligned}
\beta_{\lambda_{\phi}}^{(2, \mathrm{n})}= & \frac{1}{2 \kappa^{2}}\left[\lambda_{m}^{2}\left(24 \lambda_{m}-7 \lambda_{\sigma}\right)+\lambda_{\phi}\left(-14 \lambda_{m}^{2}+16 \lambda_{m} \lambda_{\sigma}\right.\right. \\
& \left.\left.-3 \lambda_{\sigma}^{2}\right)+\lambda_{\phi}^{2}\left(-80 \lambda_{m}+6 \lambda_{\sigma}\right)\right], \\
\beta_{\lambda_{m}}^{(2, \mathrm{n})}= & -\frac{\lambda_{m}}{6 \kappa^{2}}\left(48 \lambda_{\phi} \lambda_{m}+6 \lambda_{\phi} \lambda_{\sigma}+123 \lambda_{m}^{2}\right. \\
& \left.+86 \lambda_{m} \lambda_{\sigma}+3 \lambda_{\sigma}^{2}\right), \\
\beta_{\lambda_{\sigma}}^{(2, \mathrm{n})}= & -\frac{1}{2 \kappa^{2}}\left(48 \lambda_{m}^{3}+4 \lambda_{m}^{2} \lambda_{\sigma}+21 \lambda_{\sigma}^{3}\right), \\
\beta_{\lambda_{6}}^{(2, \mathrm{n})}= & \frac{1}{4 \kappa^{2}} \lambda_{\phi} \lambda_{m}\left(7 \lambda_{\phi}-14 \lambda_{m}+\lambda_{\sigma}\right), \\
\beta_{\lambda_{8}}^{(2, \mathrm{n})}= & \frac{1}{2 \kappa^{2}} \lambda_{\phi} \lambda_{m}^{2} .
\end{aligned}
$$

Here $\beta_{\lambda}^{(2, \mathrm{n})}$, which appears for each $\lambda$ at two loops is the mentioned correction, which is absent if this theory is regularized with $\mu=$ constant, when one breaks explicitly the scale symmetry. Notice that $\lambda_{6,8}$ also run in this order in the scale-invariant theory.

We conclude that from the two-loop running of the couplings, encoded by the beta functions, one can distinguish between the theory with (spontaneously broken) scale symmetry at quantum level and that in which this symmetry is broken explicitly by quantum corrections (with $\mu=$ constant). There is a simple way to understand this difference: the theory regularized with $\mu=$ constant, and two fields $\phi, \sigma$ is renormalizable while our model, scale invariant at quantum level, is non-renormalizable. This is due to the scaleinvariant non-polynomial terms of type $\phi^{6} / \sigma^{2}, \phi^{8} / \sigma^{4}$ generated at one- and two-loop level. ${ }^{7}$ This justifies the different beta functions in the two approaches starting from the twoloop level. This is an interesting result of the paper.

\footnotetext{
7 This non-renormalizability argument is different from that in [6] which does not apply here; see [8].
}

\subsection{Two-loop potential after renormalization}

Finally, we present the two-loop potential $U$ after renormalization. It has the form

$U=\underbrace{V+V^{(1)}+V^{(1, \mathrm{n})}}_{=U_{1}}+V^{(2)}+V^{(2, \mathrm{n})}$

where $U_{1}$ is the one-loop result of (13). $V^{(2)}$ is a two-loop correction identical to that obtained in the theory regularized with $\mu=$ constant (up to replacing $\mu \rightarrow z \sigma$ ), while $V^{(2, n)}$ are new two-loop terms that involve derivatives of $\mu(\sigma)$ w.r.t. $\sigma$ (similar to the one-loop $\left.V^{(1, \mathrm{n})}\right) .{ }^{8}$ The long expressions of $V^{(2)}, V^{(2, n)}$ are given in Appendix B, Eq. (B.5). $U$ contains new, non-polynomial effective operators, such as $\phi^{6} / \sigma^{2}$ and $\phi^{8} / \sigma^{4}$, etc.:

$U=\frac{7 \lambda_{\phi}^{3}}{576 \kappa^{2}} \frac{\phi^{8}}{\sigma^{4}}+\frac{5 \lambda_{\phi}^{3}}{24 \kappa^{2}} \frac{\phi^{6}}{\sigma^{2}}+\cdots$

All non-polynomial terms present in the potential can be expanded about the ground state

$\phi=\langle\phi\rangle+\delta \phi, \quad \sigma=\langle\sigma\rangle+\delta \sigma$

where $\delta \phi$ and $\delta \sigma$ represent fluctuations about the ground state. Then each non-polynomial operator becomes an infinite series expansion about the point $\langle\phi\rangle /\langle\sigma\rangle$. For example

$$
\begin{aligned}
\frac{\phi^{6}}{\sigma^{2}} & =(\langle\phi\rangle+\delta \phi)^{4} \frac{\langle\phi\rangle^{2}}{\langle\sigma\rangle^{2}}\left(1+\frac{2 \delta \phi}{\langle\phi\rangle}+\frac{\delta \phi^{2}}{\langle\phi\rangle^{2}}+\cdots\right) \\
& \times\left(1-\frac{2 \delta \sigma}{\langle\sigma\rangle}+\frac{3 \delta \sigma^{2}}{\langle\sigma\rangle^{2}}+\cdots\right)
\end{aligned}
$$

and similarly for the operator $\phi^{8} / \sigma^{4}$ in $U$, etc. Although we did not present the ground state of the one-loop potential, this is well known to satisfy the relation $\langle\phi\rangle^{2} /\langle\sigma\rangle^{2}=$ $-3 \lambda_{m} / \lambda_{\sigma}(1+$ loop corrections) [8]. Using this information in Eqs. (39) and (37), one sees that in the classical decoupling limit $\lambda_{m} \rightarrow 0$, the non-polynomial operators of (37) do vanish.

It is important to stress that only operators of the form $\phi^{2 n+4} / \sigma^{2 n}, n \geq 1$ were generated in the two-loop potential, but no operator like $\sigma^{2 n+4} / \phi^{2 n}, n \geq 1$ is present. This is due to the way the subtraction function enters in the loop corrections, via derivatives w.r.t. $\sigma$ of $\mu(\sigma)^{\epsilon}$ which are suppressed by positive powers of $\mu(\sigma)$. This means that all higher dimensional operators are ultimately suppressed by (large) $\langle\sigma\rangle$ and not proportional to it. This is welcome for the hierarchy problem, since such terms could otherwise lead to corrections to the Higgs mass of the type $\lambda_{\phi}^{3}\langle\sigma\rangle^{2}$ requiring tuning the Higgs self-coupling $\lambda_{\phi}$, and thus re-introducing the hierarchy problem. This problem is avoided at least at one loop $[5,8]$.

\footnotetext{
8 See [24-26] for further discussion of the Goldstone modes contributions to the potential.
} 


\section{Two-loop Callan-Symanzik for the potential}

A good check of our two-loop scale-invariant potential is the Callan-Symanzik equation, in its version for scale-invariant theories [10]. This equation states the independence of the two-loop potential of the subtraction (dimensionless) parameter $z$; this parameter fixes the subtraction scale to $z\langle\sigma\rangle$, after spontaneous scale symmetry breaking. The equation is

$$
\frac{\mathrm{d} U}{\mathrm{~d} \ln z}=\left(z \frac{\partial}{\partial z}+\beta_{\lambda_{j}} \frac{\partial}{\partial \lambda_{j}}-\phi \gamma_{\phi} \frac{\partial}{\partial \phi}-\sigma \gamma_{\sigma} \frac{\partial}{\partial \sigma}\right) U(\lambda, z)=0
$$

where the $j$-summation runs over $\lambda_{j}=\lambda_{\phi}, \lambda_{m}, \lambda_{\sigma}, \lambda_{6}, \lambda_{8}$. Equation (40) can be re-written as a set of equations at a given order of $\lambda$ 's (or number of loops). To help one trace the difference between our scale-invariant result and that of the same theory but with $\mu=$ constant, below we use for $U$ the decomposition given in Eq. (36) while for the beta functions we write

$\beta_{\lambda_{j}}=\beta_{\lambda_{j}}^{(1)}+\beta_{\lambda_{j}}^{(2)}+\beta_{\lambda_{j}}^{(2, \mathrm{n})}$.

The terms in the beta function correspond to one loop $\left(\beta_{\lambda}^{(1)}\right)$, and two loops only $\left(\beta_{\lambda}^{(2)}\right)$ and two-loop new parts $\left(\beta_{\lambda}^{(2, \mathrm{n})}\right)$. Then, with the two-loop anomalous dimensions $\gamma_{\phi}$, $\gamma_{\sigma}$ defined after Eq. (30), a careful analysis shows that Eq. (40) splits into

$$
\begin{aligned}
& \frac{\partial V^{(1)}}{\partial \ln z}+\beta_{\lambda_{j}}^{(1)} \frac{\partial V}{\partial \lambda_{j}}=0, \\
& \frac{\partial V^{(1, \mathrm{n})}}{\partial \ln z}=0, \\
& \frac{\partial V^{(2)}}{\partial \ln z}+\left(\beta_{\lambda_{j}}^{(2)} \frac{\partial}{\partial \lambda_{j}}-\gamma_{\phi} \phi \frac{\partial}{\partial \phi}-\gamma_{\sigma} \sigma \frac{\partial}{\partial \sigma}\right) V \\
& \quad+\beta_{\lambda_{j}}^{(1)} \frac{\partial V^{(1)}}{\partial \lambda_{j}}=0, \\
& \frac{\partial V^{(2, n)}}{\partial \ln z}+\beta_{\lambda_{j}}^{(2, \mathrm{n})} \frac{\partial V}{\partial \lambda_{j}}+\beta_{\lambda_{j}}^{(1)} \frac{\partial V^{(1, \mathrm{n})}}{\partial \lambda_{j}}=0,
\end{aligned}
$$

where $V$ includes the new terms ${ }^{9}\left(\lambda_{6} / 6\right) \phi^{6} / \sigma^{2}+\left(\lambda_{8} / 8\right)$ $\phi^{8} / \sigma^{4}$. We checked that these equations are respected. Eqs. (42), (44) express the usual Callan-Symanzik equation (of the theory with $\mu=$ constant), whereas (43) and (45) constitute a new part, which is nonzero only when $\mu=\mu(\sigma)$. Equation (43) is obvious and hardly revealing. But checking Eq. (45) is more difficult. For this one also needs to take account of the "new" corrections to two-loop beta functions of $\lambda_{6,8}$, see Eq. (35), and also the $z$-dependent part of $V^{(2, \mathrm{n})}$ which we write

\footnotetext{
${ }^{9}$ Although $\lambda_{5}, \lambda_{6}$ were set to 0 at the tree level, they have non-zero beta functions and since in the Callan-Symanzik equation the couplings are replaced by running couplings, these terms are present in V.
}

$$
\begin{aligned}
V^{(2, \mathrm{n})}= & \frac{1}{192 \kappa^{2}}\left[\overline{\ln } V_{p}+\overline{\ln } V_{m}\right]\left[\left(-144 \lambda_{\phi}^{2} \lambda_{m}-111 \lambda_{\phi} \lambda_{m}^{2}\right.\right. \\
& \left.-168 \lambda_{m}^{3}+9 \lambda_{\phi}^{2} \lambda_{\sigma}-40 \lambda_{m}^{2} \lambda_{\sigma}\right) \phi^{4} \\
& -\left(192 \lambda_{\phi} \lambda_{m}^{2}+705 \lambda_{m}^{3}+37 \lambda_{\phi} \lambda_{m} \lambda_{\sigma}\right. \\
& \left.+368 \lambda_{m}^{2} \lambda_{\sigma}+106 \lambda_{m} \lambda_{\sigma}^{2}\right) \phi^{2} \sigma^{2} \\
& -\left(48 \lambda_{m}^{3}+46 \lambda_{m}^{2} \lambda_{\sigma}+63 \lambda_{\sigma}^{3}\right) \sigma^{4}+\left(18 \lambda_{\phi}^{2} \lambda_{m}\right. \\
& \left.-24 \lambda_{\phi} \lambda_{m}^{2}+3 \lambda_{m}^{3}+3 \lambda_{\phi} \lambda_{m} \lambda_{\sigma}\right) \frac{\phi^{6}}{\sigma^{2}} \\
& \left.+3 \lambda_{\phi} \lambda_{m}^{2} \frac{\phi^{8}}{\sigma^{4}}\right]+z \text {-independent terms, where } \\
& \overline{\ln } A \equiv \ln \frac{A}{(z \sigma)^{2} 4 \pi e^{-\gamma_{E}}}-1,
\end{aligned}
$$

where $\gamma_{E}=0.5772 \ldots$ Here $V_{p}$ and $V_{m}$ are field dependent eigenvalues of the matrix of second derivatives $V_{i j}$ w.r.t. $i, j=\phi, \sigma$ of the tree-level potential. Given this, the CallanSymanzik equation of the potential is verified at the two-loop level.

\section{Conclusions}

Quantum scale invariance with spontaneous breaking may provide a solution to the cosmological constant and the hierarchy problem. The "traditional" method for loop calculations breaks explicitly classical scale symmetry of a theory due to the regularization which introduces a subtraction scale (DR scale, cut-off, Pauli-Villars scale). However, it is well known how to perform quantum calculations in a manifestly scale-invariant way: the subtraction scale is replaced by a subtraction function of the field(s) (dilaton $\sigma$ ) which when acquiring a VEV spontaneously, generates this scale $\mu(\langle\sigma\rangle)=z\langle\sigma\rangle$. The Goldstone mode of this symmetry is the dilaton field which remains a flat direction of the quantum scale-invariant potential.

Starting with a classically scale-invariant action, we computed the two-loop scalar potential of $\phi$ (Higgs-like) and $\sigma$ in a scale-invariant regularization. The one- and twoloop potential are scale invariant and contain new terms beyond the usual corrections obtained for $\mu=$ constant (Coleman-Weinberg, etc.), due to field derivatives of $\mu(\sigma)$. They also contain interesting effective non-polynomial operators $\phi^{6} / \sigma^{2}$ and $\phi^{8} / \sigma^{4}$, etc., allowed by scale symmetry, showing that such theories are non-renormalizable. These operators can be expanded about the non-zero $\langle\phi\rangle$ and $\langle\sigma\rangle$, to obtain an infinite series of effective polynomial ones, suppressed by $\langle\sigma\rangle \gg\langle\phi\rangle$ (such hierarchy can be enforced by one initial, classical tuning of the couplings). The nonpolynomial operators emerge from evanescent interactions $(\propto \epsilon)$ between $\phi$ and $\sigma$ that vanish in $d=4$ but are required by scale invariance in $d=4-2 \epsilon$. Previous work also showed 
that the Higgs mass is stable against quantum corrections at one loop, $m_{\phi}^{2} \ll\langle\sigma\rangle^{2}$, which we expect to remain true beyond this order because only spontaneous scale symmetry breaking is present.

We checked the consistency of the two-loop scaleinvariant potential by showing that it satisfies the CallanSymanzik equation in its scale-invariant formulation. To this purpose we computed the two-loop beta functions of the couplings of the theory. While one-loop beta functions are exactly those of the same theory of $\phi, \sigma$ regularized with $\mu=$ constant, the two-loop beta functions differ from those of the theory regularized with explicit breaking of scale symmetry ( $\mu=$ constant). In conclusion, the running of the couplings enables one to distinguish between spontaneous and explicit breaking of quantum scale symmetry of the action.

Acknowledgements The work of D. Ghilencea was supported by a grant from Romanian National Authority for Scientific Research (CNCS-UEFISCDI) under Project Number PN-II-ID-PCE-2011-30607. The work of P. Olszewski and Z. Lalak was supported by the Polish NCN Grants DEC-2012/04/A/ST2/00099 and 2014/13/N/ST2/ 02712 .

Open Access This article is distributed under the terms of the Creative Commons Attribution 4.0 International License (http://creativecomm ons.org/licenses/by/4.0/), which permits unrestricted use, distribution, and reproduction in any medium, provided you give appropriate credit to the original author(s) and the source, provide a link to the Creative Commons license, and indicate if changes were made.

Funded by SCOAP ${ }^{3}$.

\section{Appendix A: Coefficients of the counterterms}

Assuming $\lambda_{n}=0, n \geqslant 6$ at tree level, the coefficients of Eqs. (29) and (33) are

$$
\begin{aligned}
& \delta_{1}^{\phi}=-\frac{3}{2}\left(\lambda_{\phi}^{2}+\lambda_{m}^{2}+2 \frac{\lambda_{m}^{3}}{\lambda_{\phi}}\right), \\
& \delta_{1}^{m}=-\frac{1}{4}\left(\lambda_{\phi}^{2}+6 \lambda_{\phi} \lambda_{m}+10 \lambda_{m}^{2}+6 \lambda_{m} \lambda_{\sigma}+\lambda_{\sigma}^{2}\right), \\
& \delta_{1}^{\sigma}=-\frac{3}{2}\left(2 \frac{\lambda_{m}^{2}}{\lambda_{\sigma}}+\lambda_{m}^{2}+\lambda_{\sigma}^{2}\right), \\
& \nu_{1}^{\phi}=\frac{1}{8}\left[\frac{\lambda_{m}^{2}}{\lambda_{\phi}}\left(24 \lambda_{m}-7 \lambda_{\sigma}\right)-\left(14 \lambda_{m}^{2}-16 \lambda_{m} \lambda_{\sigma}+3 \lambda_{\sigma}^{2}\right)\right. \\
&\left.-\lambda_{\phi}\left(80 \lambda_{m}-6 \lambda_{\sigma}\right)\right],
\end{aligned}
$$

$\nu_{1}^{m}=-\frac{1}{24}\left[123 \lambda_{m}^{2}+86 \lambda_{m} \lambda_{\sigma}+3 \lambda_{\sigma}^{2}+6 \lambda_{\phi}\left(8 \lambda_{m}+\lambda_{\sigma}\right)\right]$,

$\nu_{1}^{\sigma}=-\frac{1}{8 \lambda_{\sigma}}\left(48 \lambda_{m}^{3}+4 \lambda_{m}^{2} \lambda_{\sigma}+21 \lambda_{\sigma}^{3}\right)$,

$\nu_{1}^{6}=\frac{1}{16} \frac{\lambda_{\phi} \lambda_{m}}{\lambda_{6}}\left(7 \lambda_{\phi}-14 \lambda_{m}+\lambda_{\sigma}\right)$,

$$
\begin{aligned}
v_{1}^{8} & =\frac{1}{8} \frac{\lambda_{\phi} \lambda_{m}^{2}}{\lambda_{8}} \\
\delta_{2}^{\phi} & =\frac{3}{4}\left[3 \lambda_{\phi}^{2}+4 \lambda_{m}^{2}+\frac{\lambda_{m}^{2}}{\lambda_{\phi}}\left(4 \lambda_{m}+\lambda_{\sigma}\right)\right], \\
\delta_{2}^{m} & =\frac{1}{4}\left(2 \lambda_{\phi}^{2}+6 \lambda_{\phi} \lambda_{m}+\lambda_{\phi} \lambda_{\sigma}+19 \lambda_{m}^{2}+6 \lambda_{m} \lambda_{\sigma}+2 \lambda_{\sigma}^{2}\right),
\end{aligned}
$$

$$
\delta_{2}^{\sigma}=\frac{3}{4}\left[\frac{\lambda_{m}^{2}}{\lambda_{\sigma}}\left(\lambda_{\phi}+4 \lambda_{m}\right)+4 \lambda_{m}^{2}+3 \lambda_{\sigma}^{2}\right]
$$

\section{Appendix B: The finite part of the two-loop potential}

We provide here the finite part of the two-loop potential, $V^{(2)}+V^{(2, n)}$ of Eqs. (25) and (36). This is rather long, we thus use a simplified notation. The propagators are found from: $\left(\widetilde{D}_{p}\right)_{i j}=p^{2} \delta_{i j}-\widetilde{V}_{i j}$. To simplify the calculation it helps to write them as

$$
\begin{gathered}
\left(\widetilde{D}^{-1}\right)_{i j}=\frac{\tilde{a}_{i j}}{p^{2}-\widetilde{V}_{p}}+\frac{\tilde{b}_{i j}}{p^{2}-\widetilde{V}_{m}}, \quad \tilde{b}_{i j}=\delta_{i j}-\tilde{a}_{i j} \\
\tilde{a}_{11}=\tilde{b}_{22}=\frac{\widetilde{V}_{p}-\widetilde{V}_{22}}{\widetilde{V}_{p}-\widetilde{V}_{m}}, \quad \tilde{a}_{22}=\tilde{b}_{11}=1-\tilde{a}_{11} \\
=\frac{\widetilde{V}_{p}-\widetilde{V}_{11}}{\widetilde{V}_{p}-\widetilde{V}_{m}}, \quad \tilde{a}_{12}=\tilde{a}_{21}=\frac{\widetilde{V}_{12}}{\widetilde{V}_{p}-\widetilde{V}_{m}},
\end{gathered}
$$

where $\widetilde{V}_{p}, \widetilde{V}_{m}$ are the field-dependent eigenvalues of matrix $\widetilde{V}_{i j}=\partial^{2} \widetilde{V} / \partial s_{i} \partial s_{j}, i, j=1,2 ; s_{1}=\phi, s_{2}=\sigma$, and $\widetilde{V}=$ $\mu(\sigma)^{2 \epsilon} V$ where $V$ is the tree-level potential in $d=4$. We introduce the following coefficients (without ${ }^{\sim}$ ) of the Taylor expansions in $\epsilon$ (see Appendix $C$ for their values in terms of the couplings and fields):

$\tilde{a}_{i j}=a_{i j}+\epsilon a_{i j}^{1}+\epsilon^{2} a_{i j}^{2}+\mathcal{O}\left(\epsilon^{3}\right), \quad b_{i j}=\delta_{i j}-a_{i j}$,

$b_{i j}^{1}=-a_{i j}^{1}, \quad b_{i j}^{2}=-a_{i j}^{2}$

$\widetilde{V}_{i j k \ldots}=\mu(\sigma)^{2 \epsilon}\left[v_{i j k \ldots}+\epsilon u_{i j k \ldots}+\epsilon^{2} w_{i j k \ldots}+\mathcal{O}\left(\epsilon^{3}\right)\right]$,

where:

$\widetilde{V}_{i j k \ldots}=\frac{\partial^{4} \widetilde{V}}{\partial s_{i} \partial s_{j} \partial s_{k} \ldots}, \quad v_{i j k \ldots}=\frac{\partial^{4} V}{\partial s_{i} \partial s_{j} \partial s_{k} \cdots}$,

$i, j, k, \ldots=1,2 ; \quad s_{1}=\phi, s_{2}=\sigma$,

$\widetilde{V}_{p}=\mu(\sigma)^{2 \epsilon} V_{p}\left[1+c_{p}^{1} \epsilon+c_{p}^{2} \epsilon^{2}+\mathcal{O}\left(\epsilon^{3}\right)\right]$

$\widetilde{V}_{m}=\mu(\sigma)^{2 \epsilon} V_{m}\left[1+c_{m}^{1} \epsilon+c_{m}^{2} \epsilon^{2}+\mathcal{O}\left(\epsilon^{3}\right)\right]$.

Here $V_{p}$ and $V_{m}$ are the field-dependent eigenvalues of the matrix $V_{i j}$ of the tree level $V$ :

$V_{p}=1 / 2\left[V_{11}+V_{22}+\left[\left(V_{11}-V_{22}\right)^{2}+4 V_{12}^{2}\right]^{1 / 2}\right]$

with $V_{m}$ having a similar expression but with - in front of the square root. $V_{p}$ and $V_{m}$ should not be confused with derivatives $V_{i}$ of the potential. We also use the notation 
$\overline{\ln } A=\ln \frac{A}{t(z \sigma)^{2}}-1, \quad t=4 \pi e^{-\gamma_{E}}$.

Then $V^{(2)}$ and $V^{(2, n)}$ of Eqs. (25) and (36) are shown below. $V^{(2)}$ is that of the theory regularized with $\mu=$ constant, while $V^{(2, n)}$ is a new correction. They are sums of the diagrams of Eq. (22),

$$
\begin{aligned}
V^{(2)} & =V_{2, \text { old }}^{a}+V_{2, \text { old }}^{b}+V_{2, \text { old }}^{c} \\
V^{(2, \mathrm{n})} & =V_{2, n}^{a}+V_{2, n}^{b}+V_{2, n}^{c}
\end{aligned}
$$

where $a, b, c$, label the sunset ( $a$ ), snowman $(b)$, counterterm (c) diagrams, respectively. Then, in terms of the above coefficients, one finds for the sunset diagram $(a)$ :

$$
\begin{aligned}
V_{2, n}^{a}= & \frac{1}{4 \kappa^{2}}\left\{\left[v_{i j} w_{l m n}+\frac{1}{2} u_{i j k} u_{l m n}\right]\left[V_{p} a_{i l}+V_{m} b_{i l}\right]\left[a_{j m}+b_{j m}\right]\left[a_{k n}+b_{k n}\right]\right. \\
& +v_{i j k} u_{l m n}\left[\left(V_{p}\left[a_{i l}\left(1-2 \overline{\ln } V_{m}+c_{p}^{1}\right)+a_{i l}^{1}\right]+V_{m}\left[b_{i l}\left(1-2 \overline{\ln } V_{m}+c_{m}^{1}\right)+b_{i l}^{1}\right]\right)\left(a_{j m}+b_{j m}\right)\right. \\
& \left.+2\left[V_{p} a_{i l}+V_{m} b_{i l}\right]\left[a_{j m}^{1}+b_{j m}^{1}\right]\right]\left[a_{k n}+b_{k n}\right]+v_{i j k} v_{l m n}\left[\frac { 1 } { 2 } \left(V _ { p } \left[a_{i l}\left(c_{p}^{2}-c_{p}^{1}\right)+a_{i l}^{1} c_{p}^{1}\right.\right.\right. \\
& \left.\left.-2 \overline{\ln } V_{p}\left(a_{i l} c_{p}^{1}+a_{i l}^{1}\right)\right]+V_{m}\left[b_{i l}\left(c_{m}^{2}-c_{m}^{1}\right)+b_{i l}^{1} c_{m}^{1}-2 \overline{\ln } V_{m}\left(b_{i l} c_{m}^{1}+b_{i l}^{1}\right)\right]\right)\left(a_{j m}+b_{j m}\right) \\
& \left.+\left(V_{p} a_{i l}\left[c_{p}^{1}-2 \overline{\ln } V_{p}\right]+V_{m} b_{i l}\left[c_{m}^{1}-2 \overline{\ln } V_{m}\right]\right)\left(a_{j m}^{1}+b_{j m}^{1}\right)\right]\left[a_{k n}+b_{k n}\right] \\
& +v_{i j k} v_{l m n}\left[V _ { p } \left(b_{i l}\left[2 a_{j m}^{1} a_{k n}^{1}+a_{j m}^{2} b_{k n}+a_{j m}^{1} b_{k n}+2 a_{j m}^{1} b_{k n}^{1}\right]+a_{i l}\left[3 a_{j m}^{1} a_{k n}^{1}+4 a_{j n}^{2} b_{k n}\right.\right.\right. \\
& \left.\left.+2 b_{j m} b_{k n}^{1}+4 a_{j m}^{1}\left(b_{k n}+b_{k n}^{1}\right)+2 b_{j m} b_{k n}^{2}\right]+a_{i l} a_{j m}\left[3\left(a_{k n}^{1}+a_{k n}^{2}\right)+2\left(b_{k n}^{1}+b_{k n}^{2}\right)\right]\right) \\
& +V_{m}\left(a_{i l}\left[2 b_{j m}^{1} b_{k n}^{1}+b_{j m}^{2} a_{k n}+b_{j m}^{1} a_{k n}+2 b_{j m}^{1} a_{k n}^{1}\right]+b_{i l}\left[3 b_{j m}^{1} b_{k n}^{1}+4 b_{j n}^{2} a_{k n}+2 a_{j m} a_{k n}^{1}\right.\right. \\
& \left.\left.\left.\left.+4 b_{j m}^{1}\left(a_{k n}+a_{k n}^{1}\right)+2 a_{j m} a_{k n}^{2}\right]+b_{i l} b_{j m}\left[3\left(b_{k n}^{1}+b_{k n}^{2}\right)+2\left(a_{k n}^{1}+a_{k n}^{2}\right)\right]\right)\right]\right\}
\end{aligned}
$$

and

$$
\begin{aligned}
V_{2, \text { old }}^{a}= & \frac{1}{4 \kappa^{2}} v_{i j k} v_{l m n}\left\{\left[V_{p} a_{i l} \overline{\ln } V_{p}+V_{m} b_{i l} \overline{\ln } V_{m}\right]\left[a_{j m} a_{k n} \overline{\ln } V_{p}+b_{j m} b_{k n} \overline{\ln } V_{m}\right]\right. \\
& +2\left[v_{p} a_{i l} \overline{\ln }^{2} V_{p}+V_{m} b_{i l} \overline{\ln }^{2} V_{m}\right] a_{j m} b_{k n}+\frac{1}{2}\left[V_{p} b_{i l}-V_{m} a_{i l}\right]\left[\overline{\ln }^{2} V_{p}-\overline{\ln }^{2} V_{m}\right] a_{j m} b_{k n} \\
& -\left[V_{p} a_{i l} \overline{\ln } V_{p}+V_{m} b_{i l} \overline{\ln } V_{m}\right]\left[a_{j m}+b_{j m}\right]\left[a_{k n}+b_{k n}\right] \\
& +\left[V_{p} a_{i l}+V_{m} b_{i l}\right]\left[a_{j m}+b_{j m}\right]\left[a_{k n}+b_{k n}\right]\left[\frac{3}{2}+\frac{\pi^{2}}{12}\right] \\
& -\left[V_{p}\left(2 a_{i l} \Phi_{p, m}-\frac{1}{2} b_{i l} \Phi_{m, p}\right)+V_{m}\left(2 b_{i l} \Phi_{m, p}-\frac{1}{2} a_{i l} \Phi_{p, m}\right)\right] a_{j m} b_{k n} \\
& \left.-\frac{1}{3}\left[V_{p} a_{i l} a_{j m} a_{k n}+V_{m} b_{i l} b_{j m} b_{k n}\right] C\right\}
\end{aligned}
$$

with [22]

$$
\begin{aligned}
& \Phi_{p, m}= \begin{cases}\sqrt{\frac{y_{p m}}{y_{p m}-1}}\left[-4 \mathrm{Li}_{2}\left(\frac{1-\eta_{p m}}{2}\right)+2 \ln ^{2} \frac{1-\eta_{p m}}{2}-\ln ^{2} 4 y_{p m}+\frac{\pi^{2}}{3}\right], & y_{p m}>1, \\
4 \sqrt{\frac{y_{p m}}{1-y_{p m}}} \mathrm{Cl}_{2}\left(\arcsin \sqrt{y_{p m}}\right), & 1>y_{p m} \geqslant 0,\end{cases} \\
& y_{p m}=V_{m} / V_{p}, \quad \eta_{p m}=\left(1-1 / y_{p m}\right)^{1 / 2}, C=-2 \sqrt{3} \mathrm{Cl}_{2}(\pi / 3) \cong 3.5, \\
& \operatorname{Li}_{2}(\xi)=-\int_{0}^{1} \mathrm{~d} t \frac{\ln (1-\xi t)}{t}, \mathrm{Cl}_{2}(\theta)=-\int_{0}^{\theta} \mathrm{d} \theta \ln \left|2 \sin \frac{\theta}{2}\right| .
\end{aligned}
$$


Further, for the snowman diagram $(b)$ :

$$
\begin{aligned}
V_{2, n}^{b}= & \frac{1}{8 \kappa^{2}}\left\{w_{i j k l}\left(a_{i j} V_{p}+b_{i j} V_{m}\right)\left(a_{k l} V_{p}+b_{k l} V_{m}\right)-2 u_{i j k l}\left[\left(a_{i j} V_{p}+b_{i j} V_{m}\right)\right.\right. \\
& \left.\times\left(a_{k l} V_{p} \overline{\ln } V_{p}+b_{k l} V_{m} \overline{\ln } V_{m}-\left[\left(a_{k l} c_{p 1}+a_{k l}^{1}\right) V_{p}+\left(b_{k l} c_{p 1}+b_{k l}^{1}\right) V_{m}\right]\right)\right] \\
& -2 v_{i j k l}\left[2 a_{i j}\left(a_{k l} c_{p 1}+a_{k l}^{1}\right) V_{p}^{2} \overline{\ln } V_{p}+2 b_{i j}\left(b_{k l} c_{m 1}+b_{k l}^{1}\right) V_{m}^{2} \overline{\ln } V_{m}\right. \\
& +\left[a_{i j}\left(b_{k l} c_{m 1}+b_{k l}^{1}\right)+b_{i j}\left(a_{k l} c_{p 1}+a_{k l}^{1}\right)\right] V_{p} V_{m}\left(\overline{\ln } V_{p}+\overline{\ln } V_{m}\right)-\left(a_{i j} V_{p}+b_{i j} V_{m}\right) \\
& \times\left(\left[a_{k l}\left(c_{p 2}-c_{p 1}\right)+a_{k l}^{1} c_{p 1}+a_{k l}^{2}\right] V_{p}+\left[b_{k l}\left(c_{m 2}-c_{m 1}\right)+b_{k l}^{1} c_{m 1}+b_{k l}^{2}\right] V_{m}\right) \\
& \left.\left.-\frac{1}{2}\left[\left(a_{i j} c_{p 1}+a_{i j}^{1}\right) V_{p}+\left(b_{i j} c_{m 1}+b_{i j}^{1}\right) V_{m}\right]\left[\left(a_{k l} c_{p}^{1}+a_{k l}^{1}\right) V_{p}+\left(b_{k l} c_{m 1}+b_{k l}^{1}\right) V_{m}\right]\right]\right\},
\end{aligned}
$$

where $i, j, k, l=1,2$. Also

$$
\begin{aligned}
& V_{2, \text { old }}^{b}=\frac{1}{8 \kappa^{2}} v_{i j k l}\left\{\left[V_{p} a_{i j}+V_{m} b_{i j}\right]\left[V_{p} a_{k l}+V_{m} b_{k l}\right]\right. \\
& \times\left[1+\frac{\pi^{2}}{6}\right]+V_{p} V_{m} a_{i j} b_{k l}\left[\overline{\ln } V_{p}-\overline{\ln } V_{m}\right]^{2} \\
& +2\left[V_{p} a_{i j} \overline{\ln } V_{p}+V_{m} b_{i j} \overline{\ln } V_{m}\right] \\
& \left.\times\left[V_{p} a_{k l} \overline{\ln } V_{p}+V_{m} b_{k l} \overline{\ln } V_{m}\right]\right\} .
\end{aligned}
$$

For the final "counter-term" diagram $(c)$ we need to introduce the coefficients $\delta v_{i j}, \delta u_{i j}, \delta w_{i j}$ whose values will be presented shortly (Appendix C). From Eq. (11)

$\delta V_{1}=\frac{1}{\varepsilon \kappa} \mu^{2 \epsilon}\left[\delta_{0}^{\phi} \frac{\lambda_{\phi}}{4 !} \phi^{4}+\delta_{0}^{m} \frac{\lambda_{m}}{4} \phi^{2} \sigma^{2}+\delta_{0}^{\sigma} \frac{\lambda_{\sigma}}{4 !} \sigma^{4}\right]$, then

$\left(\delta V_{1}\right)_{i j}=\frac{1}{\varepsilon \kappa} \mu^{2 \epsilon}\left[\delta v_{i j}+\epsilon \delta u_{i j}+\epsilon^{2} \delta w_{i j}\right]$,

where $\left(\delta V_{1}\right)_{i j}=\partial^{2}\left(\delta V_{1}\right) / \partial s_{i} \partial s_{j}, i, j=1,2, s_{1}, s_{2}=\phi, \sigma$. With this notation, we find for diagram $(c)$

$$
\begin{aligned}
& V_{2, n}^{c}=\frac{1}{2 \kappa^{2}}\left\{\delta w_{i j}\left[a_{i j} V_{p}+b_{i j} V_{m}\right]\right. \\
& +\delta u_{i j}\left[V_{p}\left(a_{i j}\left[c_{p}^{1}-\overline{\ln } V_{p}\right]+a_{i j}^{1}\right)\right. \\
& \left.+V_{m}\left(b_{i j}\left[c_{m}^{1}-\overline{\ln } V_{m}\right]+b_{i j}^{1}\right)\right] \\
& -\delta v_{i j}\left[V_{p} \overline{\ln } V_{p}\left(a_{i j} c_{p}^{1}+a_{i j}^{1}\right)+V_{m} \overline{\ln } V_{m}\left(b_{i j} c_{m}^{1}+b_{i j}^{1}\right)\right. \\
& -V_{p}\left(a_{i j} c_{p}^{2}+\left[a_{i j}^{1}-a_{i j}\right] c_{p}^{1}+a_{i j}^{2}\right) \\
& \left.\left.-V_{m}\left(b_{i j} c_{m}^{2}+\left[b_{i j}^{1}-b_{i j}\right] c_{m}^{1}+b_{i j}^{2}\right)\right]\right\}
\end{aligned}
$$

and

$$
\begin{aligned}
V_{2, \text { old }}^{c}= & \frac{1}{4 \kappa^{2}} \delta v_{i j}\left[V_{p} a_{i j}\left(\overline{\ln }^{2} V_{p}+1+\frac{\pi^{2}}{6}\right)\right. \\
& \left.+V_{m} b_{i j}\left(\overline{\ln }^{2} V_{m}+1+\frac{\pi^{2}}{6}\right)\right] .
\end{aligned}
$$

\section{Appendix C: Coefficients entering the two-loop potential}

Below we provide the expressions of the various coefficients introduced in the r.h.s. of Eqs. (B.2) and (B.13) and used in Appendix B. The coefficients $v_{i j k l}, v_{i j k}$ are functions of $\lambda$ 's and fields

$$
\begin{aligned}
& {\left[\begin{array}{lll}
v_{1111} & u_{1111} & w_{1111} \\
v_{1112} & u_{1112} & w_{1112} \\
v_{1122} & u_{1122} & w_{1122} \\
v_{1222} & u_{1222} & w_{1222} \\
v_{2222} & u_{2222} & w_{2222}
\end{array}\right]} \\
& =\left[\begin{array}{lll}
\lambda_{\phi} & 0 & 0 \\
0 & 2 \lambda_{\phi} \frac{\phi}{\sigma} & 2 \lambda_{\phi} \frac{\phi}{\sigma} \\
\lambda_{m} & 3 \lambda_{m}-\lambda_{\phi} \frac{\phi^{2}}{\sigma^{2}} & \lambda_{\phi} \frac{\phi^{2}}{\sigma^{2}}+5 \lambda_{m} \\
0 & \frac{2}{3} \lambda_{\phi} \frac{\phi^{3}}{\sigma^{3}}+2 \lambda_{m} \frac{\phi}{\sigma} & 8 \lambda_{m} \frac{\phi}{\sigma}-\frac{4}{3} \lambda_{\phi} \frac{\phi^{3}}{\sigma^{3}} \\
\lambda_{\sigma} & -\frac{1}{2} \lambda_{\phi} \frac{\phi^{4}}{\sigma^{4}}+\frac{25}{6} \lambda_{\sigma}-\lambda_{m} \frac{\phi^{2}}{\sigma^{2}} & \frac{4}{3} \lambda_{\phi} \frac{\phi^{4}}{\sigma^{4}}+10 \lambda_{\sigma}-2 \lambda_{m} \frac{\phi^{2}}{\sigma^{2}}
\end{array}\right]
\end{aligned}
$$

and

$$
\begin{aligned}
{\left[\begin{array}{lll}
v_{111} & u_{111} & w_{111} \\
v_{112} & u_{112} & w_{112} \\
v_{122} & u_{122} & w_{122} \\
v_{222} & u_{222} & w_{222}
\end{array}\right] } \\
=\left[\begin{array}{lll}
\lambda_{\phi} \phi & 0 & 0 \\
\lambda_{m} \sigma & \lambda_{\phi} \frac{\phi^{2}}{\sigma}+\sigma \lambda_{m} & \lambda_{\phi} \frac{\phi^{2}}{\sigma}+\lambda_{m} \sigma \\
\lambda_{m} \phi & 3 \lambda_{m} \phi-\frac{1}{3} \lambda_{\phi} \frac{\phi^{3}}{\sigma^{2}} & \frac{1}{3} \lambda_{\phi} \frac{\phi^{3}}{\sigma^{2}}+5 \lambda_{m} \phi \\
\lambda_{\sigma} \sigma & \frac{1}{6} \lambda_{\phi} \frac{\phi^{4}}{\sigma^{3}}+\frac{13}{6} \lambda_{\sigma} \sigma+\lambda_{m} \frac{\phi^{2}}{\sigma}-\frac{1}{3} \lambda_{\phi} \frac{\phi^{4}}{\sigma^{3}}+\frac{11}{3} \lambda_{\sigma} \sigma+4 \lambda_{m} \frac{\phi^{2}}{\sigma}
\end{array}\right] .
\end{aligned}
$$

Further, the coefficients $\delta v_{i j}, \delta u_{i j}$ and $\delta w_{i j}$ of Eq. (B.13) are

$$
\begin{aligned}
\delta v_{11} & =\frac{1}{4 \kappa}\left[3\left(\lambda_{\phi}^{2}+\lambda_{m}^{2}\right) \phi^{2}+\lambda_{m}\left(\lambda_{\phi}+4 \lambda_{m}+\lambda_{\sigma}\right) \sigma^{2}\right] \\
\delta u_{11} & =\delta w_{11}=0 \\
\delta v_{12} & =\frac{1}{2 \kappa} \lambda_{m}\left(\lambda_{\phi}+4 \lambda_{m}+\lambda_{\sigma}\right) \phi \sigma
\end{aligned}
$$




$$
\begin{aligned}
& \delta u_{12}=\delta w_{12}=\frac{1}{2 \kappa}\left[\left(\lambda_{\phi}^{2}+\lambda_{m}^{2}\right) \phi^{2}\right. \\
& \left.+\lambda_{m}\left(\lambda_{\phi}+4 \lambda_{m}+\lambda_{\sigma}\right) \sigma^{2}\right] \frac{\phi}{\sigma}, \\
& \delta v_{22}=\frac{1}{4 \kappa^{2}}\left[\left(4 \lambda_{m}^{2}+\lambda_{\phi} \lambda_{m}+\lambda_{m} \lambda_{\sigma}\right) \phi^{2}+3\left(\lambda_{m}^{2}+\lambda_{\sigma}^{2}\right) \sigma^{2}\right], \\
& \delta u_{22}=\frac{1}{8 \kappa^{2}} \frac{1}{\sigma^{2}}\left[-\left(\lambda_{\phi}^{2}+\lambda_{m}^{2}\right) \phi^{4}\right. \\
& \left.+6 \lambda_{m}\left(\lambda_{\phi}+4 \lambda_{m}+\lambda_{\sigma}\right) \phi^{2} \sigma^{2}+7\left(\lambda_{\sigma}^{2}+\lambda_{m}^{2}\right) \sigma^{4}\right], \\
& \delta w_{22}=\frac{1}{8 \kappa^{2}} \frac{1}{\sigma^{2}}\left[\left(\lambda_{\phi}^{2}+\lambda_{m}^{2}\right) \phi^{4}\right. \\
& \left.+10 \lambda_{m}\left(\lambda_{\phi}+4 \lambda_{m}+\lambda_{\sigma}\right) \phi^{2} \sigma^{2}+9\left(\lambda_{\sigma}^{2}+\lambda_{m}^{2}\right) \sigma^{4}\right] .
\end{aligned}
$$

and

$$
\begin{aligned}
S^{2}= & \frac{1}{4}\left[\left(\lambda_{\phi}-\lambda_{m}\right)^{2} \phi^{4}+2\left(\lambda_{\phi} \lambda_{m}+7 \lambda_{m}^{2}-\lambda_{\phi} \lambda_{\sigma}\right.\right. \\
& \left.\left.+\lambda_{m} \lambda_{\sigma}\right) \phi^{2} \sigma^{2}+\left(\lambda_{m}-\lambda_{\sigma}\right)^{2} \sigma^{4}\right],
\end{aligned}
$$

while $V_{m}$ and $V_{p}$ (see also (B.3)) are

$$
\left[\begin{array}{l}
V_{p} \\
V_{m}
\end{array}\right]=\frac{1}{4}\left[\begin{array}{l}
\left(\lambda_{\sigma}+\lambda_{m}\right) \sigma^{2}+\left(\lambda_{\phi}+\lambda_{m}\right) \phi^{2}+2 S \\
\left(\lambda_{\sigma}+\lambda_{m}\right) \sigma^{2}+\left(\lambda_{\phi}+\lambda_{m}\right) \phi^{2}-2 S
\end{array}\right] .
$$

For $c_{p}^{2}, c_{m}^{2}$ (with the above $V_{p}$ and $V_{m}$ )

$$
\left[\begin{array}{l}
V_{p} c_{p}^{2} \\
V_{m} c_{m}^{2}
\end{array}\right]=\frac{1}{24} \frac{1}{\sigma^{2}}\left[\begin{array}{l}
\lambda_{\phi} \phi^{4}+30 \lambda_{m} \phi^{2} \sigma^{2}+9 \lambda_{\sigma} \sigma^{4}+R_{2} \\
\lambda_{\phi} \phi^{4}+30 \lambda_{m} \phi^{2} \sigma^{2}+9 \lambda_{\sigma} \sigma^{4}-R_{2}
\end{array}\right],
$$

where

$$
\begin{aligned}
R_{2}= & \frac{1}{\sigma^{2} S^{3}}\left[\sigma^{2} \phi^{10} \lambda_{\phi}\left(13 \lambda_{\phi}^{3}+27 \lambda_{\phi} \lambda_{m}^{2}-39 \lambda_{\phi}^{2} \lambda_{m}+3 \lambda_{m}^{3}\right)+\sigma^{4} \phi^{8}\left(-23 \lambda_{\sigma} \lambda_{\phi}^{3}\right.\right. \\
& \left.+3 \lambda_{\phi} \lambda_{m}^{2}\left[3 \lambda_{\sigma}+16 \lambda_{\phi}\right]+5 \lambda_{\phi}^{2} \lambda_{m}\left[6 \lambda_{\sigma}+25 \lambda_{\phi}\right]-423 \lambda_{\phi} \lambda_{m}^{3}+90 \lambda_{m}^{4}\right) \\
& +\sigma^{6} \phi^{6}\left(\lambda_{\sigma} \lambda_{\phi}^{2}\left[7 \lambda_{\sigma}-27 \lambda_{\phi}\right]+3 \lambda_{m}^{3}\left[99 \lambda_{\sigma}+151 \lambda_{\phi}\right]+\lambda_{\phi} \lambda_{m}^{2}\left[553 \lambda_{\phi}-615 \lambda_{\sigma}\right]\right. \\
& \left.+\lambda_{\sigma} \lambda_{\phi} \lambda_{m}\left[9 \lambda_{\sigma}+65 \lambda_{\phi}\right]+2034 \lambda_{m}^{4}\right)+\sigma^{8} \phi^{4}\left(3 \lambda_{\sigma}^{2} \lambda_{\phi}\left[\lambda_{\sigma}+27 \lambda_{\phi}\right]+3 \lambda_{m}^{3}\left[641 \lambda_{\sigma}+261 \lambda_{\phi}\right]\right. \\
& \left.+\lambda_{\sigma} \lambda_{m}^{2}\left[351 \lambda_{\sigma}-521 \lambda_{\phi}\right]-\lambda_{\sigma} \lambda_{\phi} \lambda_{m}\left[361 \lambda_{\sigma}+81 \lambda_{\phi}\right]+3438 \lambda_{m}^{4}\right)+\sigma^{10} \phi^{2}\left(-81 \lambda_{\sigma}^{3} \lambda_{\phi}\right. \\
& \left.+\lambda_{\sigma} \lambda_{m}^{2}\left[292 \lambda_{\sigma}-81 \lambda_{\phi}\right]+9 \lambda_{\sigma}^{2} \lambda_{m}\left[19 \lambda_{\sigma}+18 \lambda_{\phi}\right]-609 \lambda_{\sigma} \lambda_{m}^{3}+342 \lambda_{m}^{4}\right) \\
& \left.-27 \sigma^{12} \lambda_{\sigma}\left[\lambda_{m}-\lambda_{\sigma}\right]^{3}\right] .
\end{aligned}
$$

The coefficients $c_{p}^{1}, c_{p}^{2}, c_{m}^{1}, c_{m}^{2}$, also $V_{p}$ and $V_{m}$ introduced in Eq. (B.2) obey the expressions

$$
\begin{aligned}
& {\left[\begin{array}{l}
V_{p} c_{p}^{1} \\
V_{m} c_{m}^{1}
\end{array}\right]=\frac{1}{24} \frac{1}{\sigma^{2}}} \\
& \quad \times\left[\begin{array}{l}
-\lambda_{\phi} \phi^{4}+18 \lambda_{m} \phi^{2} \sigma^{2}+7 \lambda_{\sigma} \sigma^{4}+R_{1} \\
-\lambda_{\phi} \phi^{4}+18 \lambda_{m} \phi^{2} \sigma^{2}+7 \lambda_{\sigma} \sigma^{4}-R_{1}
\end{array}\right]
\end{aligned}
$$

where

$$
\begin{aligned}
R_{1}= & \frac{1}{2 S}\left[\lambda_{\phi}\left(\lambda_{\phi}-\lambda_{m}\right) \phi^{6}+\left(15 \lambda_{\phi} \lambda_{m}-\lambda_{\phi} \lambda_{\sigma}+18 \lambda_{m}^{2}\right) \phi^{4} \sigma^{2}\right. \\
& +\left(-7 \lambda_{\phi} \lambda_{\sigma}+78 \lambda_{m}^{2}+25 \lambda_{m} \lambda_{\sigma}\right) \phi^{2} \sigma^{4} \\
& \left.+7 \lambda_{\sigma}\left(-\lambda_{m}+\lambda_{\sigma}\right) \sigma^{6}\right]
\end{aligned}
$$

Finally, $a_{i j}, a_{i j}^{1}, a_{i j}^{2}, b_{i j}, b_{i j}^{1}, b_{i j}^{2}$ introduced in (B.2) and used in Appendix B have the values

$$
\begin{aligned}
a_{11}= & 1-a_{22}=b_{22}=1-b_{11}=\frac{1}{2} \\
& +\frac{1}{4 S}\left[\lambda_{\phi} \phi^{2}+\lambda_{m}\left(-\phi^{2}+\sigma^{2}\right)-\lambda_{\sigma} \sigma^{2}\right], \\
a_{12}= & a_{21}=-b_{12}=-b_{21}=\frac{\lambda_{m} \phi \sigma}{S}, \\
a_{11}^{1}= & -a_{22}^{1}=-b_{11}^{1}=b_{22}^{1}=\frac{\lambda_{m} \phi^{2}}{6 S^{3}}\left[\lambda_{\phi}\left(-2 \lambda_{\phi}+3 \lambda_{m}\right) \phi^{4}\right. \\
& \left.+2\left(\lambda_{\phi} \lambda_{\sigma}-4 \lambda_{\phi} \lambda_{m}-6 \lambda_{m}^{2}\right) \phi^{2} \sigma^{2}-\left(6 \lambda_{m}^{2}+\lambda_{m} \lambda_{\sigma}\right) \sigma^{4}\right]
\end{aligned}
$$

with $S$ of Eq. (C.11). Also

$$
\begin{aligned}
a_{12}^{1}= & a_{21}^{1}=-b_{12}^{1}=-b_{21}^{1}=\frac{\phi}{24 \sigma S^{3}}\left[\phi^{6} \lambda_{\phi}\left(2 \lambda_{\phi}^{2}-5 \lambda_{\phi} \lambda_{m}+3 \lambda_{m}^{2}\right)\right. \\
& +\phi^{4} \sigma^{2}\left(-4 \lambda_{\sigma} \lambda_{\phi}^{2}+5 \lambda_{\phi} \lambda_{m}\left[\lambda_{\sigma}+2 \lambda_{\phi}\right]+\lambda_{\phi} \lambda_{m}^{2}-12 \lambda_{m}^{3}\right) \\
& +\phi^{2} \sigma^{4}\left(2 \lambda_{\sigma}^{2} \lambda_{\phi}+\lambda_{m}^{2}\left[14 \lambda_{\phi}-13 \lambda_{\sigma}\right]-9 \lambda_{\sigma} \lambda_{\phi} \lambda_{m}\right. \\
& \left.\left.+6 \lambda_{m}^{3}\right)+\sigma^{6} \lambda_{m}\left(-\lambda_{\sigma}^{2}-5 \lambda_{\sigma} \lambda_{m}+6 \lambda_{m}^{2}\right)\right] .
\end{aligned}
$$


Further

$$
\begin{aligned}
a_{11}^{2}= & -a_{22}^{2}=-b_{11}^{2}=b_{22}^{2}=\frac{\phi^{2}}{288 \sigma^{2} S^{5}}\left\{\phi^{10} \lambda_{\phi}^{2}\left(-4 \lambda_{\phi}^{3}-31 \lambda_{\phi} \lambda_{m}^{2}+20 \lambda_{\phi}^{2} \lambda_{m}+15 \lambda_{m}^{3}\right)\right. \\
& +\phi^{8} \sigma^{2} \lambda_{\phi}\left[12 \lambda_{\sigma} \lambda_{\phi}^{3}+\lambda_{\phi} \lambda_{m}^{2}\left[31 \lambda_{\sigma}+180 \lambda_{\phi}\right]-20 \lambda_{\phi}^{2} \lambda_{m}\left[2 \lambda_{\sigma}+3 \lambda_{\phi}\right]+5 \lambda_{\phi} \lambda_{m}^{3}-192 \lambda_{m}^{4}\right] \\
& +2 \phi^{6} \sigma^{4}\left[-6 \lambda_{\sigma}^{2} \lambda_{\phi}^{3}+\lambda_{\phi} \lambda_{m}^{3}\left[44 \lambda_{\phi}-163 \lambda_{\sigma}\right]-\lambda_{\phi}^{2} \lambda_{m}^{2}\left[19 \lambda_{\sigma}+132 \lambda_{\phi}\right]\right. \\
& \left.+2 \lambda_{\sigma} \lambda_{\phi}^{2} \lambda_{m}\left[5 \lambda_{\sigma}+28 \lambda_{\phi}\right]+450 \lambda_{\phi} \lambda_{m}^{4}+144 \lambda_{m}^{5}\right]-2 \phi^{4} \sigma^{6}\left[-2 \lambda_{\sigma}^{3} \lambda_{\phi}^{2}+\lambda_{m}^{4}\left[270 \lambda_{\phi}\right.\right. \\
& \left.\left.-96 \lambda_{\sigma}\right]+\lambda_{\phi} \lambda_{m}^{3}\left[236 \lambda_{\phi}-463 \lambda_{\sigma}\right]+\lambda_{\sigma} \lambda_{\phi} \lambda_{m}^{2}\left[71 \lambda_{\sigma}-118 \lambda_{\phi}\right]+22 \lambda_{\sigma}^{2} \lambda_{\phi}^{2} \lambda_{m}+1008 \lambda_{m}^{5}\right] \\
& +\phi^{2} \sigma^{8} \lambda_{m}\left[-8 \lambda_{\sigma}^{3} \lambda_{\phi}+12 \lambda_{m}^{3}\left[29 \lambda_{\sigma}-31 \lambda_{\phi}\right]+\lambda_{\sigma} \lambda_{m}^{2}\left[184 \lambda_{\phi}-117 \lambda_{\sigma}\right]+49 \lambda_{\sigma}^{2} \lambda_{\phi} \lambda_{m}\right. \\
& \left.\left.-468 \lambda_{m}^{4}\right]+\sigma^{10} 3 \lambda_{m}^{2}\left(-7 \lambda_{\sigma}^{3}+16 \lambda_{\sigma} \lambda_{m}^{2}+27 \lambda_{\sigma}^{2} \lambda_{m}-36 \lambda_{m}^{3}\right)\right\}
\end{aligned}
$$

Finally

$$
\begin{aligned}
a_{12}^{2}= & a_{21}^{2}=-b_{12}^{2}=-b_{21}^{2}=-\frac{\phi}{576 \sigma^{3} S^{5}}\left[-\phi^{12} \lambda_{\phi}^{2}\left(3 \lambda_{m}-2 \lambda_{\phi}\right)\left(\lambda_{m}-\lambda_{\phi}\right)^{2}\right. \\
& +2 \sigma^{2} \phi^{10} \lambda_{\phi}\left(-3 \lambda_{\phi}^{3}\left[\lambda_{\sigma}+2 \lambda_{\phi}\right]-\lambda_{\phi} \lambda_{m}^{2}\left[4 \lambda_{\sigma}+99 \lambda_{\phi}\right]+\lambda_{\phi}^{2} \lambda_{m}\left[7 \lambda_{\sigma}+57 \lambda_{\phi}\right]\right. \\
& \left.+22 \lambda_{\phi} \lambda_{m}^{3}+30 \lambda_{m}^{4}\right)+\sigma^{4} \phi^{8}\left(2 \lambda_{\sigma} \lambda_{\phi}^{3}\left[3 \lambda_{\sigma}+17 \lambda_{\phi}\right]+\lambda_{\phi} \lambda_{m}^{3}\left[160 \lambda_{\sigma}+301 \lambda_{\phi}\right]\right. \\
& \left.+2 \lambda_{\phi}^{2} \lambda_{m}^{2}\left[28 \lambda_{\sigma}+237 \lambda_{\phi}\right]-\lambda_{\phi}^{2} \lambda_{m}\left[184 \lambda_{\sigma} \lambda_{\phi}+7 \lambda_{\sigma}^{2}+84 \lambda_{\phi}^{2}\right]-972 \lambda_{\phi} \lambda_{m}^{4}-72 \lambda_{m}^{5}\right) \\
& +2 \sigma^{6} \phi^{6}\left(-\lambda_{\sigma}^{2} \lambda_{\phi}^{2}\left[\lambda_{\sigma}+15 \lambda_{\phi}\right]-6 \lambda_{m}^{4}\left[7 \lambda_{\sigma}-68 \lambda_{\phi}\right]+\lambda_{\phi} \lambda_{m}^{3}\left[379 \lambda_{\phi}-651 \lambda_{\sigma}\right]\right. \\
& \left.+\lambda_{\phi} \lambda_{m}^{2}\left[-5 \lambda_{\sigma} \lambda_{\phi}+71 \lambda_{\sigma}^{2}-108 \lambda_{\phi}^{2}\right]+\lambda_{\sigma} \lambda_{\phi}^{2} \lambda_{m}\left[13 \lambda_{\sigma}+75 \lambda_{\phi}\right]+1116 \lambda_{m}^{5}\right) \\
& +\sigma^{8} \phi^{4}\left(6 \lambda_{\sigma}^{3} \lambda_{\phi}^{2}+12 \lambda_{m}^{4}\left[92 \lambda_{\sigma}+45 \lambda_{\phi}\right]+\lambda_{m}^{3}\left[540 \lambda_{\sigma} \lambda_{\phi}+59 \lambda_{\sigma}^{2}-264 \lambda_{\phi}^{2}\right]\right. \\
& \left.-6 \lambda_{\sigma} \lambda_{\phi} \lambda_{m}^{2}\left[91 \lambda_{\sigma}-41 \lambda_{\phi}\right]+\lambda_{\sigma}^{2} \lambda_{\phi} \lambda_{m}\left[44 \lambda_{\sigma}-37 \lambda_{\phi}\right]+324 \lambda_{m}^{5}\right) \\
& +2 \sigma^{10} \phi^{2}\left(\lambda_{\sigma}^{4} \lambda_{\phi}+6 \lambda_{m}^{4}\left[31 \lambda_{\sigma}-13 \lambda_{\phi}\right]+\lambda_{\sigma} \lambda_{m}^{3}\left[89 \lambda_{\phi}-103 \lambda_{\sigma}\right]\right. \\
& \left.+\lambda_{\sigma}^{2} \lambda_{m}^{2}\left[41 \lambda_{\sigma}+8 \lambda_{\phi}\right]-20 \lambda_{\sigma}^{3} \lambda_{\phi} \lambda_{m}+72 \lambda_{m}^{5}\right) \\
& \left.+\sigma^{12}\left(-\lambda_{m}\right)\left(\lambda_{m}-\lambda_{\sigma}\right)^{2}\left(-11 \lambda_{\sigma}^{2}+24 \lambda_{\sigma} \lambda_{m}+36 \lambda_{m}^{2}\right)\right]
\end{aligned}
$$

which enter in the expression of the two-loop potential.

\section{References}

1. C. Wetterich, Cosmology and the fate of dilatation symmetry. Nucl. Phys. B 302, 668 (1988) (see for an early work)

2. I. Bars, P. Steinhardt, N. Turok, Local conformal symmetry in physics and cosmology. Phys. Rev. D 89(4), 043515 (2014). arXiv:1307.1848 [hep-th] (see for the local conformal symmetry, and references therein)

3. F. Englert, C. Truffin, R. Gastmans, Conformal invariance in quantum gravity. Nucl. Phys. B 117, 407 (1976)

4. S. Deser, Scale invariance and gravitational coupling. Ann. Phys. 59, 248 (1970)

5. M. Shaposhnikov, D. Zenhausern, Quantum scale invariance, cosmological constant and hierarchy problem. Phys. Lett. B 671, 162 (2009). arXiv:0809.3406 [hep-th]

6. M.E. Shaposhnikov, F.V. Tkachov, Quantum scale-invariant models as effective field theories. arXiv:0905.4857 [hep-th]

7. R. Armillis, A. Monin, M. Shaposhnikov, Spontaneously broken conformal symmetry: dealing with the trace anomaly. JHEP 1310, 030 (2013). arXiv:1302.5619 [hep-th]
8. D.M. Ghilencea, Manifestly scale-invariant regularization and quantum effective operators. Phys. Rev. D 93(10), 105006 (2016). arXiv:1508.00595 [hep-ph]

9. D.M. Ghilencea, One-loop potential with scale invariance and effective operators. arXiv:1508.00595 [hep-ph] (Proceedings of the 15th Hellenic School and Workshops on Elementary Particle Physics and Gravity (CORFU2015), Corfu, 1-25 Sep 2015)

10. C. Tamarit, Running couplings with a vanishing scale anomaly. JHEP 1312, 098 (2013). arXiv:1309.0913 [hep-th]

11. W.A. Bardeen, On naturalness in the standard model, FERMILABCONF-95-391-T, C95-08-27.3

12. P.G. Ferreira, C.T. Hill, G.G. Ross, Scale-independent inflation and hierarchy generation. arXiv:1603.05983 [hep-th]

13. M. Shaposhnikov, D. Zenhausern, Scale invariance, unimodular gravity and dark energy. Phys. Lett. B 671, 187 (2009). arXiv:0809.3395 [hep-th]

14. I. Oda, Higgs mechanism in scale-invariant gravity. Adv. Stud. Theor. Phys. 8, 215 (2014). arXiv:1308.4428 [hep-ph]

15. I. Oda, Conformal Higgs gravity. Adv. Stud. Theor. Phys. 9, 595 (2015). arXiv:1505.06760 [gr-qc]

16. I. Oda, Classically scale-invariant B-L model and conformal gravity. Phys. Lett. B 724, 160 (2013). doi:10.1016/j.physletb.2013.06. 014. arXiv:1305.0884 [hep-ph] 
17. A. Kobakhidze, Quantum relaxation of the Higgs mass. Eur. Phys. J. C 75(8), 384 (2015). arXiv:1506.04840 [hep-ph]

18. G.K. Karananas, M. Shaposhnikov, Scale invariant alternatives to general relativity. II. Dilaton properties. Phys. Rev. D 93(8), 084052 (2016). arXiv:1603.01274 [hep-th]

19. D. Blas, M. Shaposhnikov, D. Zenhausern, Scale-invariant alternatives to general relativity. Phys. Rev. D 84, 044001 (2011). arXiv:1104.1392 [hep-th]

20. K. Allison, C.T. Hill, G.G. Ross, Ultra-weak sector, Higgs boson mass, and the dilaton. Phys. Lett. B 738, 191 (2014). arXiv:1404.6268 [hep-ph]

21. R. Foot, A. Kobakhidze, K.L. McDonald, R.R. Volkas, Poincaré protection for a natural electroweak scale. Phys. Rev. D 89(11), 115018 (2014). arXiv:1310.0223 [hep-ph]

22. A.I. Davydychev, J.B. Tausk, Two loop selfenergy diagrams with different masses and the momentum expansion. Nucl. Phys. B 397, $123(1993)$
23. C. Ford, I. Jack, D.R.T. Jones, The standard model effective potential at two loops. Nucl. Phys. B 387, 373 (1992). arXiv:hep-ph/0111190 (erratum: Nucl. Phys. B 504, 551, 1997)

24. J.R. Espinosa, M. Garny, T. Konstandin, Interplay of infrared divergences and gauge-dependence of the effective potential. arXiv:1607.08432 [hep-ph]

25. J. Elias-Miro, J.R. Espinosa, T. Konstandin, Taming infrared divergences in the effective potential. JHEP 1408, 034 (2014). arXiv:1406.2652 [hep-ph]

26. S.P. Martin, Taming the Goldstone contributions to the effective potential. Phys. Rev. D 90(1), 016013 (2014). arXiv:1406.2355 [hep-ph] 